\title{
SPECTRAL ANALYSIS OF THE GRAM MATRIX OF MIXTURE MODELS *
}

\author{
Florent Benaych-Georges ${ }^{1}$ And Romain Couillet ${ }^{2}$
}

\begin{abstract}
This text is devoted to the asymptotic study of some spectral properties of the Gram matrix $W^{\top} W$ built upon a collection $w_{1}, \ldots, w_{n} \in \mathbb{R}^{p}$ of random vectors (the columns of $W$ ), as both the number $n$ of observations and the dimension $p$ of the observations tend to infinity and are of similar order of magnitude. The random vectors $w_{1}, \ldots, w_{n}$ are independent observations, each of them belonging to one of $k$ classes $\mathcal{C}_{1}, \ldots, \mathcal{C}_{k}$. The observations of each class $\mathcal{C}_{a}(1 \leq a \leq k)$ are characterized by their distribution $\mathcal{N}\left(0, p^{-1} C_{a}\right)$, where $C_{1}, \ldots, C_{k}$ are some non negative definite $p \times p$ matrices. The cardinality $n_{a}$ of class $\mathcal{C}_{a}$ and the dimension $p$ of the observations are such that $n_{a} / n(1 \leq a \leq k)$ and $p / n$ stay bounded away from 0 and $+\infty$. We provide deterministic equivalents to the empirical spectral distribution of $W^{\top} W$ and to the matrix entries of its resolvent (as well as of the resolvent of $W W^{\top}$ ). These deterministic equivalents are defined thanks to the solutions of a fixed-point system. Besides, we prove that $W^{\top} W$ has asymptotically no eigenvalues outside the bulk of its spectrum, defined thanks to these deterministic equivalents. These results are directly used in our companion paper [R. Couillet and F. Benaych-Georges, Electron. J. Stat. 10 (2016) 1393-1454.], which is devoted to the analysis of the spectral clustering algorithm in large dimensions. They also find applications in various other fields such as wireless communications where functionals of the aforementioned resolvents allow one to assess the communication performance across multi-user multi-antenna channels.
\end{abstract}

Mathematics Subject Classification. 60B20, 15B52, 62H30.

Received October 16, 2015. Accepted March 14, 2016.

\section{INTRODUCTION AND MAIN RESULTS}

\subsection{Introduction}

In this article, we consider the Gram matrix $W^{\top} W$, where $W=\left[w_{1}, \ldots, w_{n}\right], w_{i} \in \mathbb{R}^{p}$, is a collection of independent random vectors. Each $w_{i}$ belongs to one of $k$ classes $\mathcal{C}_{1}, \ldots, \mathcal{C}_{k}$, with $\mathcal{C}_{a}(1 \leq a \leq k)$ the class of vectors distributed as $\mathcal{N}\left(0, p^{-1 / 2} C_{a}\right)$, where $C_{1}, \ldots, C_{k}$ are some non negative definite $p \times p$ matrices.

This $k$-fold class setting comes in naturally in the field of machine learning and in statistical problems such as kernel spectral clustering (see the companion paper [10], where the present results find direct applications). Clustering algorithms are methods used to discover unknown subgroups or clusters in data: they seek partitions

\footnotetext{
Keywords and phrases. Random matrices, extreme eigenvalue statistics, mixture models, spectral clustering.

* Couillet's work is supported by the ANR Project RMT4GRAPH (ANR-14-CE28-0006).

1 MAP 5, UMR CNRS 8145, Université Paris Descartes, Paris, France. florent.benaych-georges@parisdescartes.fr

2 Centrale Supélec, LSS, Université Paris Sud, Gif sur Yvette, France. romain.couillet@supelec.fr
} 
of the data into distinct groups so that the observations within each group are the most similar to one another and the observations belonging to different groups are the most dissimilar from each other. The most commonly used such algorithms are $k$-means, the hierarchical clustering and EM [13,16]. Spectral clustering techniques (see e.g. $[19,22])$ make use of the spectrum of a similarity matrix of the data or of a more involved derived version of it (such as the associated Laplacian matrix) to perform dimensionality reduction before clustering in fewer dimensions, usually thanks to one of the previously mentioned algorithms. More specifically, given $n$ observations $x_{1}, \ldots, x_{n} \in \mathbb{R}^{p}$ one wants to cluster, one chooses a similarity measurement, such as $\kappa\left(x_{i}, x_{j}\right):=f\left(\frac{\left\|x_{j}-x_{i}\right\|^{2}}{p}\right)$, for a well chosen decreasing function $f$, and defines the similarity matrix $A:=\left[\kappa\left(x_{i}, x_{j}\right)\right]_{i, j=1}^{n}$. Then, each observation $x_{i} \in \mathbb{R}^{p}$ is replaced by its projection $y_{i}$ onto the linear span of the $k$ leading eigenvectors of the similarity matrix $A$ or of its "Laplacian" $L:=\left[\frac{\kappa\left(x_{i}, x_{j}\right)}{\sqrt{d_{i} d_{j}}}\right]_{i, j=1}^{n}$, where, for each $i, d_{i}:=\sum_{j} \kappa\left(x_{i}, x_{j}\right)$, and $k$ means (or another aforementioned standard algorithms) is then performed on $y_{1}, \ldots, y_{n}$. It appears in our companion article [10] that, when the observations $x_{i}$ belong to classes, such that $x_{i}=\mu_{a}+p^{1 / 2} w_{i}$ for some $w_{i} \sim \mathcal{N}\left(0, p^{-1} C_{a}\right)$ when falling in class, say, $\mathcal{C}_{a}^{\prime}$, the performance of the clustering algorithm relies on theoretical results on the spectrum and the resolvent of the aforementioned matrix $W^{\top} W$, some of which are stated and proved here.

Another class of applications is found in the field of wireless communications, where, letting $W_{a}=$ $\left[w_{\sum_{j=1}^{a-1} n_{j}+1}, \ldots, w_{\sum_{j=1}^{a} n_{j}}\right] \in \mathbb{R}^{p \times n_{j}}, w_{i} \sim \mathcal{N}\left(0, p^{-1} C_{a}\right)$ be the communication channel between a $p$-antenna transmitter and an $n_{a}$-antenna receiver and $\sigma^{2}$ some ambient noise variance, functionals of the type $\log \operatorname{det}\left(W W^{\top}+\sigma^{2} I_{p}\right)$ or $\operatorname{tr} W_{a}^{\top} W_{a}\left(W W^{\top}+\sigma^{2} I_{p}\right)^{-1}$ are instrumental to evaluate the maximally achievable communication rate across the channel, see e.g. [11,18].

The purpose of the present paper is to prove several technical results concerning the resolvent matrices of $W^{\top} W$ and $W W^{\top}$, which are then used, along with other arguments, to obtain a deterministic equivalent for the empirical spectral measure of $W^{\top} W$ as $n, p \rightarrow \infty$ while $n / p$ remains away from 0 and $\infty$, and to show that no eigenvalue can be found at macroscopic distance from the support of this measure. Some of these results and related questions (which we sometimes rederive for completion) can be found scattered in the literature: as in [11], where the same model is considered but only linear functionals of the empirical spectral measure of $W^{\mathrm{\top}} W$ are considered, or in $[1,9,23,24]$ where the spectral analyses of different models are considered, leading in particular to analogous equations to those introduced in Proposition 1.3 below (some of the results of [23,24] are immediate corollaries of our present results), or else in $[3-6,8,17,21]$, where the question of isolated eigenvalues of related matrix models was also considered.

\subsection{Model presentation}

Let us start by introducing some notations. In what follows, everything, when not mentioned as constant or fixed, depends implicitly on the parameters $n, p, \ldots$ introduced below.

Let $k$ be fixed, $n, p, n_{1}, \ldots, n_{k}$ be some positive integers all tending to infinity such that $n_{1}+\cdots+n_{k}=n$ and such that the ratios

$$
c_{0}=\frac{p}{n}, \quad c_{a}=\frac{n_{a}}{n} \quad(a=1, \ldots, k)
$$

all stay bounded away from 0 and $+\infty$. We also introduce some positive semi-definite $p \times p$ matrices $C_{1}, \ldots, C_{k}$ which we suppose to be bounded, in operator norm, by a constant, and some standard real Gaussian matrices

$$
Z_{1} \in \mathbb{R}^{p \times n_{1}}, \ldots, Z_{k} \in \mathbb{R}^{p \times n_{k}}
$$

and define the $p \times n$ random matrix $W$ by

$$
W:=p^{-1 / 2}\left[C_{1}^{1 / 2} Z_{1}, \ldots, C_{k}^{1 / 2} Z_{k}\right] .
$$




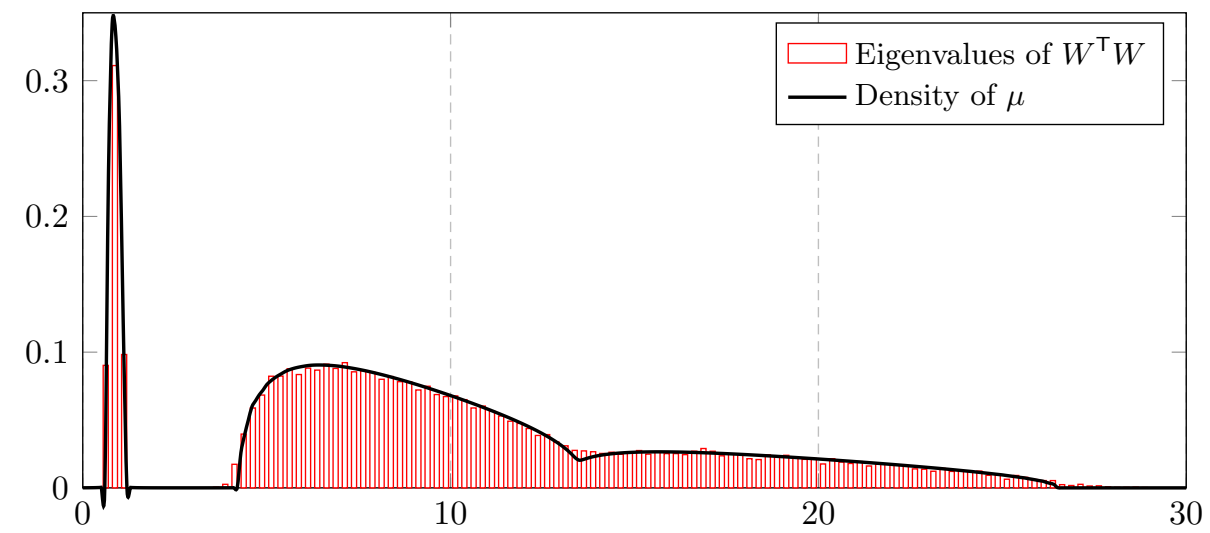

FIgURE 1. Eigenvalues of $W^{\top} W$ (across 1000 realizations) versus $\mu, n=32, p=256, k=3$, $c_{1}=1 / 8, c_{2}=5 / 8, c_{3}=1 / 4,\left[C_{a}\right]_{i j}=(8(a-1)+1)[(a-1) / 5]^{|i-j|}$. The density of $\mu$ is computed as detailed in Remark 1.4.

In what follows, we will be interested in the empirical spectral distribution $\mu_{W^{\top} W}$ of $W^{\top} W$ (i.e. the uniform probability measure on the spectrum of $W^{\top} W$ ) and in the matrix entries of the resolvents

$$
Q_{z}:=\left(W^{\top} W-z\right)^{-1} \quad \text { and } \quad \tilde{Q}_{z}:=\left(W W^{\top}-z\right)^{-1} .
$$

\subsection{Main results}

Recall that everything, when not mentioned as constant, depends implicitly on the parameters $n, p, z, \ldots$ On the contrary, a constant element is non random and independent of all other parameters. Two elements are said to be at a macroscopic distance from each other if there is a constant $\varepsilon>0$ such that there distance is lowerbounded by $\varepsilon$. We define the Stieltjes transform of a probability measure $\mu$ as the function $m_{\mu}(z):=\int \frac{\mu(\mathrm{d} t)}{t-z}$. The set of signed measures on $\mathbb{R}$ is endowed with the weak topology, i.e. the topology defined by continuous bounded functions.

Theorem 1.1. The measure $\mu$ defined by its Stieltjes transform

$$
m_{\mu}(z)=c_{0} \sum_{a=1}^{k} c_{a} g_{a}(z),
$$

where the vector $\left(g_{1}(z), \ldots, g_{k}(z)\right), z \in \mathbb{C} \backslash \mathbb{R}$, is defined by Proposition 1.3 , is a deterministic probability measure (depending on $n, p, \ldots$ ) with compact support $\mathcal{S}$ such that we have the almost sure convergences

$$
\begin{array}{r}
\mu_{W^{\top} W}-\mu \longrightarrow 0 \\
\operatorname{dist}\left(\operatorname{Spec}\left(W^{\top} W\right), \mathcal{S} \cup\{0\}\right) \longrightarrow 0 .
\end{array}
$$

Besides, if the matrices $C_{1}, \ldots, C_{k}, I_{p}$ are all positive definite and linearly independent, $m_{\mu}(z)$ extends continuously from $\mathbb{C}^{+}:=\{z \in \mathbb{C}, \Im(z)>0\}$ to $\mathbb{C}^{+} \cup \mathbb{R}^{*}$ and, except for a possible atom at zero, $\mu$ has a continuous density given by $\frac{1}{\pi} \Im\left(m_{\mu}(x)\right)\left(x \in \mathbb{R}^{*}\right)$.

Remark 1.2. We believe that the technical assumption that $C_{1}, \ldots, C_{k}, I_{p}$ are all positive definite and linearly independent to obtain a continuous limiting density can be relaxed. As it stands though, since an $\varepsilon$ perturbation of $C_{1}, \ldots, C_{k}$ can always ensure these conditions while modifying $\operatorname{Spec}\left(W^{\top} W\right)$ in a controlled manner, we have that for all large $n, p, \operatorname{Spec}\left(W^{\top} W\right)$ is close to a spectrum having continuous deterministic equivalent and still defined by the equations of Proposition 1.3 below, with some slightly perturbed versions of the matrices $C_{a}$. 
The vector $\left(g_{1}(z), \ldots, g_{k}(z)\right)$ mentioned in Theorem 1.1 is defined explicitly in the following proposition.

Proposition 1.3 (Definition of $\left.g_{1}(z), \ldots, g_{k}(z)\right)$. For any $z \in \mathbb{C} \backslash \mathbb{R}$, there is a unique vector $\left(g_{1}(z), \ldots, g_{k}(z)\right) \in$ $\mathbb{C}^{k}$ (depending on $n, p, \ldots$ ) such that for each $a$,

$$
\Im z \Im g_{a}(z) \geq 0, \quad \Im z \Im\left(z g_{a}(z)\right) \geq 0, \quad c_{0}\left|g_{a}(z)\right| \leq(\Im z)^{-1}
$$

and

$$
c_{0} g_{a}(z)=-\frac{1}{z} \frac{1}{1+\tilde{g}_{a}(z)}, \quad \tilde{g}_{a}(z)=-\frac{1}{z} \frac{1}{p} \operatorname{tr} C_{a}\left(I_{p}+\sum_{b=1}^{k} c_{b} g_{b}(z) C_{b}\right)^{-1} .
$$

Besides, the first two inequalities in (1.4) are in fact strict and the functions $c_{0} g_{a}(z)(a=1, \ldots, k)$ are the Stieltjes transforms of some $\mathbb{R}_{+}$-compactly supported probability measures $\nu_{1}, \ldots, \nu_{k}$.

Remark 1.4. It appears in the Proof of Proposition 1.3 that the functions $g_{a}$ (hence the function $m_{\mu}$, by (1.1)) can be computed numerically in a very efficient way thanks to a fixed point convergence. Then, choosing $z=x+\mathrm{i} \eta$ close to the real line, we get a good approximation of the measure $\mu$ of Theorem 1.1 as $\mu \approx \frac{1}{\pi} \Im\left(m_{\mu}(x+\mathrm{i} \eta)\right) \mathrm{d} x$.

At the core of the proof of Theorem 1.1 is the following result. For $A=A(n), B=B(n)$ some random square matrices with size $n$ tending to infinity, the notation $A \leftrightarrow B$ stands for the fact that we have the convergences in probability $\frac{1}{n} \operatorname{tr} D(A-B) \longrightarrow 0$ and $d_{1}^{\top}(A-B) d_{2} \longrightarrow 0$ for all sequence $D=D(n)$ of deterministic $n \times n$ matrices of bounded norms and all deterministic sequnces vectors $d_{i}=d_{i}(n)$ of bounded norms.

Proposition 1.5 (Deterministic equivalents). Let $\mathcal{S}:=\cup_{a=1}^{k} \operatorname{supp}\left(\nu_{a}\right)$. For any $z \in \mathbb{C}$ at macroscopic distance from $\mathcal{S} \cup\{0\}$, we have

$$
\begin{aligned}
& Q_{z} \leftrightarrow \bar{Q}_{z}:=c_{0} \operatorname{diag}\left\{g_{a}(z) 1_{n_{a}}\right\}_{a=1}^{k} \\
& \tilde{Q}_{z} \leftrightarrow \overline{\tilde{Q}}_{z}:=-\frac{1}{z}\left(I_{p}+\sum_{a=1}^{k} c_{a} g_{a}(z) C_{a}\right)^{-1} .
\end{aligned}
$$

Proposition 1.5 by itself finds immediate applications in the aforementioned area of wireless communications, where functionals of the type $-\frac{1}{p} \log Q_{x}$ or $w_{i}^{*} Q_{x} w_{i}$ are fundamental quantities to evaluate achievable communication rates [11], or in machine learning where some more involved linear statistics of the Gram matrix $X X^{\top}$, with $X=p^{-\frac{1}{2}}\left[\mu_{1} 1_{n_{1}}^{\top}, \ldots, \mu_{k} 1_{n_{k}}^{\top}\right]+W$ a Gaussian mixture sample, carries relevant information for data classification and clustering [10]. For these applications, further results such as central limit theorems [18] or higher order statistics are required. For completion, we provide below some additional results that come in handy into this scope.

Proposition 1.6 (Further deterministic equivalents). For any $z_{1}, z_{2} \in \mathbb{C}$ at macroscopic distance from $\mathcal{S} \cup\{0\}$,

$$
\begin{aligned}
& Q_{z_{1}} \mathcal{D}_{a} Q_{z_{2}} \leftrightarrow \bar{Q}_{z_{1}} \mathcal{D}_{a} \bar{Q}_{z_{2}}+\sum_{b=1}^{k} R_{a b}\left(z_{1}, z_{2}\right) \bar{Q}_{z_{1}} \mathcal{D}_{b} \bar{Q}_{z_{2}} \\
& \tilde{Q}_{z_{1}} C_{a} \tilde{Q}_{z_{2}} \leftrightarrow \overline{\tilde{Q}}_{z_{1}} C_{a} \overline{\tilde{Q}}_{z_{2}}+\sum_{b=1}^{k} R_{b a}\left(z_{1}, z_{2}\right) \overline{\tilde{Q}}_{z_{1}} C_{b} \overline{\tilde{Q}}_{z_{2}} \\
& \frac{1}{p} \tilde{Q}_{z_{1}} W \mathcal{D}_{a} W^{\top} \tilde{Q}_{z_{2}} \leftrightarrow z_{1} z_{2} c_{0} c_{a} g_{a}\left(z_{1}\right) g_{a}\left(z_{2}\right) \overline{\tilde{Q}}_{z_{1}} C_{a} \overline{\tilde{Q}}_{z_{2}}
\end{aligned}
$$

where $R\left(z_{1}, z_{2}\right)_{a b}=\frac{c_{a}}{c_{b}}\left[\left(I_{k}-\Omega\left(z_{1}, z_{2}\right)\right)^{-1} \Omega\left(z_{1}, z_{2}\right)\right]_{a b}$ with, for $1 \leq a, b \leq k$,

$$
\Omega\left(z_{1}, z_{2}\right)_{a b}=c_{0} c_{b} z_{1} g_{a}\left(z_{1}\right) z_{2} g_{a}\left(z_{2}\right) \frac{1}{p} \operatorname{tr} C_{a} \overline{\tilde{Q}}_{z_{1}} C_{b} \overline{\tilde{Q}}_{z_{2}}
$$


Although it appears only at this point, the matrix $\Omega\left(z_{1}, z_{2}\right)$ is a cornerstone of the proof of Theorem 1.1. It is in particular related to the derivative of the $g_{a}(z)$ 's introduced in Proposition 1.3 as follows

$$
\left\{g_{a}^{\prime}(z)\right\}_{a=1}^{k}=c_{0}\left(I_{k}-\Omega(z, z)\right)^{-1}\left\{g_{a}^{2}(z)\right\}_{a=1}^{k} .
$$

\section{Proofs: Preliminary approximation lemma}

Here, we denote the Hadamard product (i.e. entry-wise product) of matrices or vectors by $\odot$. We shall also use both superscript notations $(\cdot)^{\top}$ and $(\cdot)^{*}$ to denote transpose and Hermitian transpose of matrices and vectors.

Lemma 2.1. Let $z, \underline{z} \in \mathbb{C} \backslash \mathbb{R}$ and $\left(g_{a}\right)_{a=1}^{k},\left(\underline{g}_{a}\right)_{a=1}^{k} \in \mathbb{C}^{k}$ such that for each $a$,

$$
\Im(z) \Im\left(g_{a}\right) \geq 0, \quad \Im(z) \Im\left(z g_{a}\right) \geq 0, \quad c_{0}\left|g_{a}\right| \leq|\Im z|^{-1}
$$

(and the same for $\left.\underline{g}_{a}, \underline{z}\right)$ and $\left(\varepsilon_{a}\right)_{a=1}^{k},\left(\underline{\varepsilon}_{a}\right)_{a=1}^{k},\left(\eta_{a}\right)_{a=1}^{k},\left(\underline{\eta}_{a}\right)_{a=1}^{k} \in \mathbb{C}^{k}$ such that for each $a=1, \ldots, k$, we have

$$
c_{0} g_{a}=\frac{1}{-z\left(1+\frac{1}{p} \operatorname{tr} C_{a} \overline{\tilde{Q}}+\eta_{a}\right)}+c_{0} \varepsilon_{a} ; \quad c_{0} \underline{g}_{a}=\frac{1}{-\underline{z}\left(1+\frac{1}{p} \operatorname{tr} C_{a} \underline{\tilde{Q}}+\underline{\eta}_{a}\right)}+c_{0} \underline{\varepsilon}_{a},
$$

with

$$
\overline{\tilde{Q}}:=-z^{-1}\left(I_{p}+\sum_{a=1}^{k} c_{a} g_{a} C_{a}\right)^{-1} ; \quad \underline{\tilde{Q}}:=-\underline{z}^{-1}\left(I_{p}+\sum_{a=1}^{k} c_{a} \underline{g}_{a} C_{a}\right)^{-1}
$$

Then:

(i) We have

$$
(I-\Omega)(\underline{g}-g)=c_{0}(\underline{z}-z+\underline{z} \underline{\eta}-z \eta) \odot(\underline{g}-\underline{\varepsilon}) \odot(g-\varepsilon)+\underline{\varepsilon}-\varepsilon
$$

for

$$
\Omega=\Omega(z, \underline{z}, g, \underline{g}, \varepsilon, \underline{\varepsilon}, \eta, \underline{\eta}):=\left[c_{0} z \underline{z}\left(\underline{g}_{a}-\underline{\varepsilon}_{a}\right)\left(g_{a}-\varepsilon_{a}\right) c_{b} \frac{1}{p} \operatorname{tr} C_{a} \overline{\tilde{Q}} C_{b} \underline{\tilde{Q}}\right]_{a, b=1}^{k} .
$$

(ii) For $C:=\max _{a}\left\|C_{a}\right\|$, we have

$$
\rho(\Omega) \leq 1-\min \left\{\frac{(\Im z)^{2}}{|z|(|\Im z|+C)}, \frac{(\Im \underline{z})^{2}}{|\underline{z}|(|\Im \underline{z}|+C)}\right\}^{2}+\|\varepsilon\|_{\infty}+\|\underline{\varepsilon}\|_{\infty}+c_{0}\left(|z|\|\eta\|_{\infty}+|\underline{z}|\|\underline{\eta}\|_{\infty}\right) .
$$

(iii) There are $P, Q$ some polynomials with non negative coefficients and $c_{1}>0$ such that for $\alpha:=$ $\max \left\{\|\varepsilon\|_{\infty},\|\underline{\varepsilon}\|_{\infty},\|\eta\|_{\infty},\|\underline{\eta}\|_{\infty}\right\}$, we have

$$
\alpha \leq c_{1} \min \left\{|\Im z|^{4},|\Im \underline{z}|^{4},|z|^{-3},|\underline{z}|^{-3}\right\} \Longrightarrow\|\underline{g}-g\| \leq(|\underline{z}-z|+\alpha) P(|z|+|\underline{z}|) Q\left(|\Im z|^{-1}+|\Im \underline{z}|^{-1}\right) .
$$

Proof. Note first that by the hypotheses $z g_{a}, \underline{z} \underline{g}_{a} \in \mathbb{C}^{+}$, we have that $\|\overline{\tilde{Q}}\| \leq|\Im z|^{-1}$. It follows that

$$
\left|c_{0} g_{a}\right| \geq|z|^{-1} \frac{1}{1+C|\Im z|^{-1}+\left|\eta_{a}\right|},
$$

for $C=\max _{a}\left\|C_{a}\right\|_{\infty}$. The same kind of inequalities hold for $\underline{\tilde{Q}}$ and $\underline{g}_{a}$. 
We have

$$
\begin{aligned}
c_{0}\left(\underline{g}_{a}-g_{a}\right) & =c_{0}\left(\underline{\varepsilon}_{a}-\varepsilon_{a}\right)+c_{0}\left(\underline{g}_{a}-\underline{\varepsilon}_{a}\right)-c_{0}\left(g_{a}-\varepsilon_{a}\right) \\
& =c_{0}\left(\underline{\varepsilon}_{a}-\varepsilon_{a}\right)+\frac{1}{z\left(1+\frac{1}{p} \operatorname{tr} C_{a} \overline{\tilde{Q}}+\eta_{a}\right)}-\frac{1}{\underline{z}\left(1+\frac{1}{p} \operatorname{tr} C_{a} \underline{\tilde{Q}}+\underline{\eta}_{a}\right)} \\
& =c_{0}\left(\underline{\varepsilon}_{a}-\varepsilon_{a}\right)+\frac{\underline{z}-z+\underline{z}_{a}-z \eta_{a}+\frac{1}{p} \operatorname{tr}\left(C_{a}(\underline{z} \underline{\tilde{Q}}-z \underline{\tilde{Q}})\right)}{z \underline{z}\left(1+\frac{1}{p} \operatorname{tr} C_{a} \overline{\tilde{Q}}+\eta_{a}\right)\left(1+\frac{1}{p} \operatorname{tr} C_{a} \underline{\tilde{Q}}+\underline{\eta}_{a}\right)} .
\end{aligned}
$$

Now, one has to notice that as $\underline{\tilde{Q}}^{-1}+\underline{z} I_{p}=-\underline{z} \sum_{b=1}^{k} c_{b} \underline{g}_{b} C_{b}$, we have

$$
\begin{aligned}
z \operatorname{tr} C_{a} \overline{\tilde{Q}}+z \underline{z} \operatorname{tr} C_{a} \overline{\tilde{Q}} \underline{\overline{\tilde{Q}}} & =z \operatorname{tr} C_{a} \overline{\tilde{Q}}\left(\underline{\tilde{\tilde{Q}}}^{-1}+\underline{z}\right) \underline{\tilde{\tilde{Q}}} \\
& =-z \underline{z} \sum_{b=1}^{k} c_{b} \underline{g}_{b} \operatorname{tr} C_{a} \overline{\tilde{Q}} C_{b} \underline{\overline{\tilde{Q}}} .
\end{aligned}
$$

In the same way, as $\overline{\tilde{Q}}^{-1}+z I_{p}=-z \sum_{b=1}^{k} c_{b} g_{b} C_{b}$, we have

$$
\begin{aligned}
\underline{z} \operatorname{tr} C_{a} \underline{\overline{\tilde{Q}}}+z \underline{z} \operatorname{tr} C_{a} \overline{\tilde{Q}} \underline{\bar{Q}} & =z \operatorname{tr} C_{a} \overline{\tilde{Q}}\left(\overline{\tilde{Q}}^{-1}+z\right) \underline{\overline{\tilde{Q}}} \\
& =-z \underline{z} \sum_{b=1}^{k} c_{b} g_{b} \operatorname{tr} C_{a} \overline{\tilde{Q}} C_{b} \underline{\tilde{Q}} .
\end{aligned}
$$

It follows that

$$
\begin{aligned}
\operatorname{tr}\left(C_{a}(\underline{z} \underline{\tilde{Q}}-z \overline{\tilde{Q}})\right) & =\underline{z} \operatorname{tr} C_{a} \underline{\tilde{\tilde{Q}}}+z \underline{z} \operatorname{tr} C_{a} \underline{\overline{\tilde{Q}}} \underline{\bar{Q}}-\left(z \operatorname{tr} C_{a} \overline{\tilde{Q}}+z \underline{z} \operatorname{tr} C_{a} \underline{\tilde{Q}} \underline{\tilde{Q}}\right) \\
& =z \underline{z} \sum_{b=1}^{k} c_{b} \underline{g}_{b} \operatorname{tr} C_{a} \overline{\tilde{Q}} C_{b} \underline{\tilde{\tilde{Q}}}-z \underline{z} \sum_{b=1}^{k} c_{b} g_{b} \operatorname{tr} C_{a} \overline{\tilde{Q}} C_{b} \underline{\overline{\tilde{Q}}} \\
& =z \underline{z} \sum_{b=1}^{k} c_{b}\left(\underline{g}_{b}-g_{b}\right) \operatorname{tr} C_{a} \overline{\tilde{Q}} C_{b} \underline{\tilde{\tilde{Q}}}
\end{aligned}
$$

and that

$$
\begin{aligned}
c_{0}\left(\underline{g}_{a}-g_{a}\right)= & c_{0}\left(\underline{\varepsilon}_{a}-\varepsilon_{a}\right)+\frac{\underline{z}-z+\underline{z}_{a}-z \eta_{a}+\frac{1}{p} \operatorname{tr}\left(C_{a}(\underline{z} \underline{\tilde{Q}}-z \underline{\tilde{Q}})\right)}{z \underline{z}\left(1+\frac{1}{p} \operatorname{tr} C_{a} \tilde{\tilde{Q}}+\eta_{a}\right)\left(1+\frac{1}{p} \operatorname{tr} C_{a} \underline{\tilde{Q}}+\underline{\eta}_{a}\right)} \\
= & c_{0}\left(\underline{\varepsilon}_{a}-\varepsilon_{a}\right)+c_{0}^{2}\left(\underline{g}_{a}-\underline{\varepsilon}_{a}\right)\left(g_{a}-\varepsilon_{a}\right) \\
& \times\left(\underline{z}_{a}-z \eta_{a}+(\underline{z}-z)+z \underline{z} \sum_{b=1}^{k} c_{b}\left(\underline{g}_{b}-g_{b}\right) \frac{1}{p} \operatorname{tr} C_{a} \overline{\tilde{Q}} C_{b} \underline{\tilde{Q}}\right) .
\end{aligned}
$$

We directly deduce (i).

To prove (ii), let us first treat the case where $\underline{z}=z^{*}$ and $\underline{\varepsilon}_{a}=\varepsilon_{a}^{*}, \underline{g}_{a}=g_{a}^{*}$ for each $a$. If $\Im(z)>0$ (the other case can be treated in the same way), it is easy to see that $\Omega$ has positive entries (as $\operatorname{tr} C_{a} \overline{\tilde{Q}} C_{b} \underline{\tilde{Q}}=$ $\left.\operatorname{tr} C_{a}^{1 / 2} \overline{\tilde{Q}} C_{b}^{1 / 2}\left(C_{a}^{1 / 2} \overline{\tilde{Q}} C_{b}^{1 / 2}\right)^{*}\right)$ and that we have, by (i),

$$
(I-\Omega) \Im(g)=c_{0} \Im(z)|g-\varepsilon|^{2}+\underbrace{\frac{c_{0}}{2}(\underline{\eta} \underline{z}-\eta z)+\frac{1}{2}(\underline{\varepsilon}-\varepsilon)}_{:=\kappa} .
$$


Thus $\kappa \in \mathbb{R}^{k}$ and if $u=\left(u_{1}, \ldots, u_{k}\right)$ is a left eigenvector of $\Omega$ associated with $\rho(\Omega)$ such that for all $a, u_{a} \geq 0$ and $\sum_{a=1}^{k} u_{a}=1$ (as per Lemma C.1 in the appendix), then we have

$$
(1-\rho(\Omega)) \sum_{a=1}^{k} u_{a} \Im\left(g_{a}\right)=c_{0} \Im(z) \sum_{a=1}^{k} u_{a}\left|g_{a}-\varepsilon_{a}\right|^{2}+u^{\top} \kappa,
$$

which implies, using successively (2.1) and (2.2),

$$
\begin{aligned}
1-\rho(\Omega) & =c_{0} \frac{\sum_{a=1}^{k} u_{a}\left|g_{a}\right|^{2}}{\sum_{a=1}^{k} u_{a} \Im\left(c_{0} g_{a}\right)(\Im(z))^{-1}}+u^{\top} \kappa \\
& \geq c_{0}^{2} \frac{\sum_{a=1}^{k} u_{a}\left|g_{a}\right|^{2}}{\sum_{a=1}^{k} u_{a}(\Im(z))^{-2}}+u^{\top} \kappa \\
& =\left(c_{0} \Im(z)\right)^{2} \sum_{a=1}^{k} u_{a}\left|g_{a}\right|^{2}+u^{\top} \kappa \\
& \geq\left(\frac{(\Im z)^{2}}{|z|(|\Im z|+C)}\right)^{2}+u^{\top} \kappa,
\end{aligned}
$$

so that the spectrum of $\Omega$ is contained in the ball with center 0 and radius

$$
1-\left(\frac{(\Im z)^{2}}{|z|(|\Im z|+C)}\right)^{2}+\|\varepsilon\|_{\infty}+c_{0}|z|\|\eta\|_{\infty} .
$$

To treat the general case, just use Lemmas C.2 and C.3 from the appendix and notice that

$$
\left|\operatorname{tr} C_{a} \overline{\tilde{Q}} C_{b} \underline{\tilde{\tilde{Q}}}\right|=\left|\operatorname{tr} C_{a}^{1 / 2} \overline{\tilde{Q}} C_{b}^{1 / 2} C_{b}^{1 / 2} \underline{\tilde{Q}} C_{a}^{1 / 2}\right| \leq \sqrt{\operatorname{tr} C_{a} \overline{\tilde{Q}} C_{b} \overline{\tilde{Q}} \operatorname{tr} C_{a} \underline{\tilde{Q}} C_{b} \underline{\tilde{Q}}}
$$

from which it follows that $\rho(\Omega(z, \underline{z}, \ldots)) \leq \sqrt{\rho\left(\Omega\left(z, z^{*}, \ldots\right)\right) \rho\left(\Omega\left(\underline{z}, \underline{z}^{*}, \ldots\right)\right)}$ and then use $\sqrt{A B} \leq \max (A, B)$ for $A, B>0$.

At last, (iii) follows from the formula of the inverse of a matrix in terms of the determinant and of the minors.

\section{Proof of Proposition 1.3}

\subsection{Uniqueness}

Note that for each fixed $z \in \mathbb{C} \backslash \mathbb{R}$, if there exist two vectors

$$
\left(g_{1}(z), \ldots, g_{k}(z)\right) \quad \text { and } \quad\left(\underline{g}_{1}(z), \ldots, \underline{g}_{k}(z)\right)
$$

satisfying (1.4) and the equations (1.5), then one can apply Lemma 2.1 with $\varepsilon=\eta=0$ : by (i), we get that $(I-\Omega)(\underline{g}-g)=0$, whereas by (ii) we know that $\rho(\Omega)<1$, which implies that $g=\underline{g}$.

\subsection{Existence}

Note first that one can focus on $\mathbb{C}^{+}$and then extend to $\mathbb{C} \backslash \mathbb{R}$ by the formula $g_{a}\left(z^{*}\right)=g_{a}(z)^{*}$. We shall first prove that there is a unique collection of functions of $z$ satisfying the weakened version of conditions (1.4) given by (3.1) and the equations (1.5).

Let $\mathcal{L}$ be the set of analytic functions $g: \mathbb{C}^{+} \rightarrow \mathbb{C}$ such that for all $z \in \mathbb{C}^{+}$,

$$
\Im g(z) \geq 0, \quad \Im(z g(z)) \geq 0, \quad c_{0}|g(z)| \leq|\Im z|^{-1}
$$


For $\eta>0$, we define, for $g, \underline{g} \in \mathcal{L}$,

$$
\mathrm{d}_{\mathcal{L}, \eta}(g, \underline{g}):=\sup _{\Im z \geq \eta}|\underline{g}(z)-g(z)| .
$$

By the analytic continuation principle, this is obviously a distance.

Lemma 3.1. $\mathrm{d}_{\mathcal{L}, \eta}$ is a complete distance on $\mathcal{L}$.

Proof. Note first that by Montel theorem $\mathcal{L}$ is a compact subset of the set of analytic functions on $\mathbb{C}^{+}$endowed with the topology of uniform convergence on compact sets. Let $\left(g_{n}\right)$ be a Cauchy sequence in $\left(\mathcal{L}, \mathrm{d}_{\mathcal{L}, \eta}\right)$. Then there is an analytic function $g$ defined on $\{z ; \Im z>\eta\}$ such that on $\{z ; \Im z>\eta\},\left(g_{n}\right)$ converges uniformly to $g$. Besides, any accumulation point of $\left(g_{n}\right)$ in the set of analytic functions on $\mathbb{C}^{+}$endowed with the topology of uniform convergence on compact sets coincides with $g$ on $\{z ; \Im z>\eta\}$, hence $g$ is the restriction to $\{z$; $z>\eta\}$ of an element of $\mathcal{L}$, and $\left(g_{n}\right)$ converges to $g$ in $\left(\mathcal{L}, \mathrm{d}_{\mathcal{L}, \eta}\right)$.

We denote $\mathcal{L}^{k}=\mathcal{L} \times \cdots \times \mathcal{L}$.

Lemma 3.2. Let $\Psi: \mathcal{L}^{k} \rightarrow \mathcal{L}^{k}$ be defined by $\Psi\left(g_{1}, \ldots, g_{k}\right)=\left(f_{1}, \ldots, f_{k}\right)$, where

$$
c_{0} f_{a}(z)=-\frac{1}{z-\frac{1}{p} \operatorname{tr} C_{a}\left(I_{p}+\sum_{b=1}^{k} c_{b} g_{b}(z) C_{b}\right)^{-1}}, \quad(a=1, \ldots, k) .
$$

Then $\Psi$ is well defined and admits a unique fixed point in $\mathcal{L}^{k}$.

Proof. Let us first make two remarks. First, for $C, D$ some non negative definite Hermitian matrices, $\operatorname{tr} C D=$ $\operatorname{tr} C^{1 / 2} D^{1 / 2}\left(C^{1 / 2} D^{1 / 2}\right)^{*} \geq 0$. By linear combination, if $C, D$ are only Hermitian matrices, $\operatorname{tr} C D \in \mathbb{R}$. Secondly, let $A$ be an invertible matrix such that $A=X+\mathrm{i} Y$, with $X, Y$ Hermitian matrices such that $Y$ non negative definite. Then

$$
A^{-1}=A^{-1} A^{*}\left(A^{-1}\right)^{*}=A^{-1} X\left(A^{-1}\right)^{*}-\mathrm{i} A^{-1} Y\left(A^{-1}\right)^{*}
$$

has a skew-Hermitian part which has the form i times a non positive definite matrix.

From both of these remarks, we deduce that for $g_{1}, \ldots, g_{k} \in \mathbb{C}$, we have

$$
\Im g_{1} \geq 0, \ldots, \Im g_{k} \geq 0 \Longrightarrow \Im \operatorname{tr} C_{a}\left(I_{p}+\sum_{b=1}^{k} c_{b} g_{b} C_{b}\right)^{-1} \leq 0
$$

and

$$
\Im\left(z g_{1}\right) \geq 0, \ldots, \Im\left(z g_{k}\right) \geq 0 \Longrightarrow \Im \operatorname{tr} C_{a}\left(z I_{p}+\sum_{b=1}^{k} c_{b} z g_{b} C_{b}\right)^{-1} \leq 0,
$$

so that $\Psi: \mathcal{L}^{k} \rightarrow \mathcal{L}^{k}$ is well defined.

Let now $\varepsilon>0$ such that for any $p \times p$ matrices $X, Y$,

$$
\left\|Y-I_{p}\right\|,\left\|X-I_{p}\right\| \leq \varepsilon \Longrightarrow\left\|Y^{-1}-X^{-1}\right\| \leq 2\|Y-X\| \text {. }
$$

Let $\eta_{0}>0$ be such that

$$
\sum_{b=1}^{k} c_{b} \eta_{0}^{-1}\left\|C_{b}\right\| \leq \varepsilon
$$

Now, fix $g, \underline{g} \in \mathcal{L}^{k}$ and set $f, \underline{f}:=\Psi(g), \Psi(\underline{g})$. For any $z \in \mathbb{C}^{+}$such that $\Im z \geq \eta_{0}$, and any $a=1, \ldots, k$, we have, for $G:=\sum_{b=1}^{k} c_{b} g_{b}(z) C_{b}$ and $\underline{G}:=\sum_{b=1}^{k} c_{b} g_{b}(z) C_{b}$,

$$
\begin{aligned}
\left|\underline{f}_{a}(z)-f_{a}(z)\right| & =\frac{c_{0}^{-1}}{\left|\left(z-\frac{1}{p} \operatorname{tr} \frac{C_{a}}{I_{p}+G}\right)\left(z-\frac{1}{p} \operatorname{tr} \frac{C_{a}}{I_{p}+\underline{G}}\right)\right|}\left|\frac{1}{p} \operatorname{tr}\left(C_{a}\left(\left(I_{p}+G\right)^{-1}-\left(I_{p}+\underline{G}\right)^{-1}\right)\right)\right| \\
& \leq 2 c_{0}^{-1} k(\Im z)^{-2} \max _{a}\left\|C_{a}\right\|^{2} \max _{a}\left|\underline{g}_{a}(z)-g_{a}(z)\right|
\end{aligned}
$$


We deduce that for $\eta_{0}$ large enough, if one endows $\mathcal{L}^{k}$ with the product distance defined by $\mathrm{d}_{\mathcal{L}, \eta}$, then $\Psi$ is a contraction. By the previous lemma, it allows one to conclude.

\subsection{Conclusion of the proof}

The functions $g_{1}, \ldots g_{k}$ are analytic on $\mathbb{C}^{+}$and satisfy (3.1) and equations (1.5). Using $\Im(z g(z)) \geq 0$ in (1.5), one easily gets that for each $a$,

$$
\lim _{\Im z \rightarrow+\infty} c_{0} z g_{a}(z)=-1 .
$$

This proves that the $c_{0} g_{a}$ 's are the Stieltjes transforms of some probability measures $\nu_{a}$, hence that for each $a$,

$$
\Im g_{a}(z)>0, \quad c_{0}\left|g_{a}(z)\right| \leq|\Im z|^{-1}
$$

Besides, as

$$
\Im\left(z g_{a}(z)\right) \geq 0,
$$

the $g_{a}$ 's are supported on $\mathbb{R}_{+}$, hence $\Im\left(z g_{a}(z)\right)>0$.

Now, it remains to prove that the $\nu_{a}$ 's have compact supports. For any $d, \varepsilon>0$, let $\mathcal{F}_{d, \varepsilon}$ denote the set of continuous functions $g:\{z ; \Re z \geq d, \Im z \geq 0\} \rightarrow \mathbb{C}$ such that for all $z \in \mathbb{R}, g(z) \in \mathbb{R}$ and for all $z \notin \mathbb{R}$,

$$
\Im g(z) \geq 0, \quad \Im(z g(z)) \geq 0, \quad|g(z)| \leq \varepsilon .
$$

Clearly, when endowed with the distance

$$
\mathrm{d}_{\mathcal{F}_{d, \varepsilon}}(g, \underline{g}):=\sup _{\Re z \geq d, \Im z \geq 0}|\underline{g}(z)-g(z)|,
$$

$\mathcal{F}_{d, \varepsilon}$ is a complete metric space. Let $\Phi: \mathcal{F}_{d, \varepsilon}^{k} \rightarrow \mathcal{F}_{d, \varepsilon}^{k}$ be defined by $\Phi\left(f_{1}, \ldots, f_{k}\right)=\left(h_{1}, \ldots, h_{k}\right)$, where

$$
c_{0} h_{a}(z)=-\frac{1}{z-\frac{1}{p} \operatorname{tr} \frac{C_{a}}{I_{p}+\sum_{b=1}^{k} c_{b} f_{b}(z) C_{b}}}, \quad(a=1, \ldots, k) .
$$

Then by the same kind of computations as in the proof of Lemma 3.2, one proves that for $a$ large enough and $\varepsilon$ small enough, $\Phi$ is well defined and admits a unique fixed point in $\mathcal{F}_{d, \varepsilon}^{k}$. By the pointwise uniqueness we already proved, this fixed point must coincide with the vector $\left(g_{1}, \ldots, g_{k}\right)$ on $\{z ; \Re z \geq d, \Im z>0\}$. We deduce that the functions $g_{1}, \ldots, g_{k}$ can be extended as continuous functions on $\mathbb{C}^{+} \cup[d,+\infty)$ taking real values on $[d,+\infty)$. By ([2], Thm. 2.4.3), we conclude that the measures $\nu_{1}, \ldots, \nu_{k}$ have supports contained in $[0, d]$.

\section{Proof of Proposition 1.5}

In this section we shall use the notation $u_{p}=O\left(v_{p}\right)$ for a sequence $u_{p}$ possibly depending on other parameters $\left(i, i^{\prime}, z, \ldots\right)$ such that there are some polynomials $P, Q$ with non negative coefficients such that uniformly in all parameters,

$$
u_{p} \leq v_{p} P(|z|) Q\left(|\Im z|^{-1}\right) .
$$

For $M=M_{p}$ a matrix, $M_{p}=O_{\|\cdot\|}\left(v_{p}\right)$ means that the operator norm $\|M\|$ of $M$ satisfies

$$
\|M\|=O\left(v_{p}\right) .
$$

Also, for $X$ a (possibly multidimensional) random variable, we set

$$
\stackrel{\circ}{X}:=X-\mathbb{E} X .
$$

At last, for each $a=1, \ldots, k$, we define $j_{a} \in \mathbb{R}^{n \times 1}$ as the column vector with $i$ th entry equal to 1 if $n_{1}+\cdots+$ $n_{a-1}<i \leq n_{1}+\cdots+n_{a}$ and to 0 otherwise and set $\mathcal{D}_{a}$, the diagonal $n \times n$ matrix with diagonal $j_{a}$. 


\subsection{Boundedness of $W W^{\top}$}

We shall use the following lemma, following from the fact that

$$
W W^{\top}=p^{-1} \sum_{a=1}^{k} C_{a}^{1 / 2} Z_{a} Z_{a}^{\top} C_{a}^{1 / 2}
$$

and that as is well known (e.g. by [12], Lem. 7.3), there are $t_{0}, c_{0}>0$ constant such that for all $t>0$,

$$
\mathbb{P}\left(\max _{a=1, \ldots, k} p^{-1}\left\|Z_{a} Z_{a}^{\top}\right\|>t_{0}+t\right) \leq \mathrm{e}^{c_{0} n\left(t-t_{0}\right)} .
$$

Lemma 4.1. There are $t_{0}, c_{0}>0$ constant such that for all $t>0$,

$$
\mathbb{P}\left(\max _{a=1, \ldots, k}\left\|W W^{\top}\right\|>t_{0}+t\right) \leq \mathrm{e}^{c_{0} n\left(t-t_{0}\right)} .
$$

\subsection{Loop equations}

We shall prove the following lemma in the next sections. For short, we shall denote $Q_{z}$ by $Q$.

Lemma 4.2. The matrix $\mathbb{E} Q$ is a diagonal matrix with diagonal entries that are constant along the classes, i.e. of the form $\sum_{a=1}^{k} \alpha_{a} \mathcal{D}_{a}$, with $\alpha_{a} \in \mathbb{C}^{+}$. Besides, for $z \in \mathbb{C} \backslash \mathbb{R}$,

$$
\begin{aligned}
& \mathbb{E} Q=-z^{-1}\left(I_{n}+\mathbb{E} D\right)^{-1}+O_{\|\cdot\|}\left(p^{-1}\right) \\
& \mathbb{E} \tilde{Q}=-z^{-1}\left(I_{p}+\sum_{a=1}^{k} \mathbb{E} \frac{1}{p} \operatorname{tr}\left(\mathcal{D}\left(j_{a}\right) Q\right) C_{a}\right)^{-1}+O_{\|\cdot\|}\left(p^{-1}\right)
\end{aligned}
$$

where

$$
D:=\sum_{a=1}^{k} \frac{1}{p} \operatorname{tr}\left(\tilde{Q} C_{a}\right) \mathcal{D}_{a} .
$$

The fact that the matrix $\mathbb{E} Q$ is diagonal follows from the Neumann expansion for $|z|$ large enough (and from analytic continuation for small $z$ ) and from the fact that the $Z_{a}$ 's are independent and with symmetric distribution. The fact that it is of the form given here follows from the invariance of the law of $W^{\top} W$ under conjugation by the appropriate permutation matrices (those with all cycles contained in a class).

For each $i=1, \ldots, n$, we denote by $C(i)$ the covariance matrix of the $i$ th column of $p^{1 / 2} W$, so that $C(i)=C_{a}$ if $n_{1}+\cdots+n_{a-1}<i \leq n_{1}+\cdots+n_{a-1}+n_{a}$.

\subsubsection{Computations on $Q$}

By the resolvent identity,

$$
Q=z^{-1} W^{\top} W Q-z^{-1} I_{n}
$$

so that, using Stein Lemma (see in appendix),

$$
\begin{aligned}
\mathbb{E} Q_{i i^{\prime}} & =z^{-1}\left(W^{\top} W Q\right)_{i i^{\prime}}-\delta_{i i^{\prime}} z^{-1} \\
& =-\delta_{i i^{\prime}} z^{-1}+z^{-1} \mathbb{E} \sum_{j, l} w_{j i} w_{j l} Q_{l i^{\prime}} \\
& =-\delta_{i i^{\prime}} z^{-1}+z^{-1} \sum_{j, l, m} \mathbb{E} w_{j i} w_{m i} \mathbb{E} \partial_{w_{m i}} w_{j l} Q_{l i^{\prime}} \\
& =-\delta_{i i^{\prime}} z^{-1}+(p z)^{-1} \sum_{j, l, m} C(i)_{j m}\left\{\mathbb{1}_{(m, i)=(j, l)} \mathbb{E} Q_{l i^{\prime}}-\mathbb{E} w_{j l}\left(Q\left(\left[\delta_{r i} w_{m s}+\delta_{s i} w_{m r}\right]_{r, s=1}^{n}\right) Q\right)_{l i^{\prime}}\right\}
\end{aligned}
$$


where we used the fact that $\mathbb{E} w_{j i} w_{m i}=p^{-1} C(i)_{j m}$. With $E_{i j}$ the matrix with unique non-zero entry $\left[E_{i j}\right]_{i j}=1$,

$$
\partial_{w_{m i}} Q=-Q\left(\partial_{w_{m i}} W^{\top} W\right) Q=-Q\left(E_{i m} W+W^{\top} E_{m i}\right) Q=-Q\left(\left[\delta_{r i} w_{m s}+\delta_{s i} w_{m r}\right]_{r, s=1}^{n}\right) Q
$$

from which, after replacement, we get

$$
\begin{aligned}
\mathbb{E} Q_{i i^{\prime}}= & -\delta_{i i^{\prime}} z^{-1}+(p z)^{-1} \sum_{j, l, m} C(i)_{j m}\left\{\mathbb{1}_{(m, i)=(j, l)} \mathbb{E} Q_{l i^{\prime}}-\mathbb{E} w_{j l}\left(Q\left(\left[\delta_{r i} w_{m s}+\delta_{s i} w_{m r}\right]_{r, s=1}^{n}\right) Q\right)_{l i^{\prime}}\right\} \\
= & -\delta_{i i^{\prime}} z^{-1}+(p z)^{-1} \mathbb{E} Q_{i i^{\prime}} \operatorname{tr} C(i)-(p z)^{-1} \sum_{j, l, m, s} C(i)_{j m} \mathbb{E} w_{j l} Q_{l i} w_{m s} Q_{s i^{\prime}} \\
& -(p z)^{-1} \sum_{j, l, m, r} C(i)_{j m} \mathbb{E} w_{j l} Q_{l r} w_{m r} Q_{i i^{\prime}} \\
= & -\delta_{i i^{\prime}} z^{-1}+(p z)^{-1} \mathbb{E} Q_{i i^{\prime}} \operatorname{tr} C(i)-(p z)^{-1} \sum_{j, m} C(i)_{j m} \mathbb{E}(W Q)_{j i}(W Q)_{m i^{\prime}} \\
& -(p z)^{-1} \sum_{j, m} C(i)_{j m} \mathbb{E}\left(W Q W^{\top}\right)_{j m} Q_{i i^{\prime}} \\
= & -\delta_{i i^{\prime}} z^{-1}+(p z)^{-1} \mathbb{E} Q_{i i^{\prime}} \operatorname{tr} C(i)-(p z)^{-1} \mathbb{E}\left(Q W^{\top} C(i) W Q\right)_{i i^{\prime}} \\
& -(p z)^{-1} \mathbb{E} \operatorname{tr}\left(W Q W^{\top} C(i)\right) Q_{i i^{\prime}} .
\end{aligned}
$$

Besides, it is easy to see that $W Q W^{\top}=W W^{\top} \tilde{Q}=z \tilde{Q}+I_{p}$ which entails

$$
\begin{aligned}
\mathbb{E} Q_{i i^{\prime}}= & -\delta_{i i^{\prime}} z^{-1}+(p z)^{-1} \mathbb{E} Q_{i i^{\prime}} \operatorname{tr} C(i)-(p z)^{-1} \mathbb{E}\left(Q W^{\top} C(i) W Q\right)_{i i^{\prime}} \\
& -(p z)^{-1} \mathbb{E} \operatorname{tr}((z \tilde{Q}+1) C(i)) Q_{i i^{\prime}} \\
= & -\delta_{i i^{\prime}} z^{-1}+(p z)^{-1} \mathbb{E} Q_{i i^{\prime}} \operatorname{tr} C(i)-(p z)^{-1} \mathbb{E}\left(Q W^{\top} C(i) W Q\right)_{i i^{\prime}}-p^{-1} \mathbb{E} \operatorname{tr}(\tilde{Q} C(i)) Q_{i i^{\prime}} \\
& -(p z)^{-1} \operatorname{tr}(C(i)) \mathbb{E} Q_{i i^{\prime}} \\
= & -\delta_{i i^{\prime}} z^{-1}-(p z)^{-1} \mathbb{E}\left(Q W^{\top} C(i) W Q\right)_{i i^{\prime}}-\mathbb{E} p^{-1} \operatorname{tr}(\tilde{Q} C(i)) Q_{i i^{\prime}} .
\end{aligned}
$$

In other words, if one defines

$$
M_{1}:=\frac{1}{z} \sum_{a=1}^{k} \mathbb{E}_{a} Q W^{\top} C_{a} W Q=\frac{1}{z} \sum_{a=1}^{k} \mathbb{E} \mathcal{D}_{a} W^{\top} \tilde{Q} C_{a} \tilde{Q} W,
$$

then we have, for $D$ as in $(4.5)$ and $\stackrel{\circ}{D}:=D-\mathbb{E} D$,

$$
\begin{aligned}
-z \mathbb{E} Q & =I_{n}+z \mathbb{E} D Q+z p^{-1} M_{1} \\
& =I_{n}+z \mathbb{E} D \mathbb{E} Q+z p^{-1}\left(M_{1}+p \mathbb{E} \stackrel{\circ}{D} Q\right) .
\end{aligned}
$$

Now, as clearly $\left\|M_{1}\right\|=O(1)$ and, by Lemma B.1,

$$
\|\mathbb{E} \stackrel{\circ}{D} Q\| \leq \mathbb{E}\|\stackrel{\circ}{D}\|\|Q\| \leq \frac{1}{|\Im z|} \mathbb{E}\|\stackrel{\circ}{D}\| \leq \frac{1}{|\Im z|} \sum_{a=1}^{k} \sqrt{\operatorname{Var}\left(p^{-1} \operatorname{tr}\left(\tilde{Q} C_{a}\right)\right)}=O\left(p^{-1}\right)
$$

it follows that

$$
-z \mathbb{E} Q=I_{n}+z \mathbb{E} D \mathbb{E} Q+O_{\|\cdot\|}\left(p^{-1}\right),
$$

and

which implies

$$
-z \mathbb{E} Q\left(I_{n}+\mathbb{E} D\right)=I_{n}+O_{\|\cdot\|}\left(p^{-1}\right),
$$

This proves (4.3).

$$
\mathbb{E} Q=-z^{-1}\left(I_{n}+\mathbb{E} D\right)^{-1}+O_{\|\cdot\|}\left(p^{-1}\right) .
$$




\subsubsection{Computations on $\tilde{Q}$}

By the resolvent identity, we have here $\tilde{Q}=z^{-1} W W^{\top} \tilde{Q}-z^{-1} I_{p}$ so that, by Stein Lemma again,

$$
\begin{aligned}
\mathbb{E} \tilde{Q}_{i i^{\prime}} & =z^{-1}\left(W W^{\top} \tilde{Q}\right)_{i i^{\prime}}-\delta_{i i^{\prime}} z^{-1} \\
& =-\delta_{i i^{\prime}} z^{-1}+z^{-1} \mathbb{E} \sum_{j, l} w_{i j} w_{l j} \tilde{Q}_{l i^{\prime}} \\
& =-\delta_{i i^{\prime}} z^{-1}+z^{-1} \sum_{j, l, m} \mathbb{E} w_{i j} w_{m j} \mathbb{E} \partial_{w_{m j}} w_{l j} \tilde{Q}_{l i^{\prime}} \\
& =-\delta_{i i^{\prime}} z^{-1}+(p z)^{-1} \sum_{j, l, m} C(j)_{i m}\left\{\mathbb{1}_{(m, j)=(l, j)} \mathbb{E} \tilde{Q}_{l i^{\prime}}-\mathbb{E} w_{l j}\left(\tilde{Q}\left(\left[\delta_{r m} w_{s j}+\delta_{s m} w_{r j}\right]_{r, s=1}^{p}\right) \tilde{Q}\right)_{l i^{\prime}}\right\}
\end{aligned}
$$

where we used the fact that $\mathbb{E} w_{i j} w_{m j}=p^{-1} C(j)_{i m}$ and that

$$
\partial_{w_{m j}} \tilde{Q}=-\tilde{Q}\left(\partial_{w_{m j}} W W^{\top}\right) \tilde{Q}=-\tilde{Q}\left(E_{m j} W^{\top}+W E_{j m}\right) \tilde{Q}=-\tilde{Q}\left(\left[\delta_{r m} w_{s j}+\delta_{s m} w_{r j}\right]_{r, s=1}^{p}\right) \tilde{Q}
$$

We get, somewhat similarly as previously

$$
\begin{aligned}
\mathbb{E} \tilde{Q}_{i i^{\prime}}= & -\delta_{i i^{\prime}} z^{-1}+(p z)^{-1} \sum_{j} \mathbb{E}(C(j) \tilde{Q})_{i i^{\prime}}-(p z)^{-1} \sum_{j, l, m, s} C(j)_{i m} \mathbb{E} w_{l j} \tilde{Q}_{l m} w_{s j} \tilde{Q}_{s i^{\prime}} \\
& -(p z)^{-1} \sum_{j, l, m, r} C(j)_{i m} \mathbb{E} w_{l j} \tilde{Q}_{l r} w_{r j} \tilde{Q}_{m i^{\prime}} \\
= & -\delta_{i i^{\prime}} z^{-1}+(p z)^{-1} \sum_{j} \mathbb{E}(C(j) \tilde{Q})_{i i^{\prime}}-(p z)^{-1} \sum_{a=1}^{k} \mathbb{E}\left(C_{a} \tilde{Q} W \mathcal{D}\left(j_{a}\right) W^{\top} \tilde{Q}\right)_{i i^{\prime}} \\
& -(p z)^{-1} \sum_{a=1}^{k} \mathbb{E}\left(C_{a} \tilde{Q}\right)_{i i^{\prime}} \operatorname{tr}\left(W \mathcal{D}\left(j_{a}\right) W^{\top} \tilde{Q}\right) \\
= & -\delta_{i i^{\prime}} z^{-1}+z^{-1} \sum_{a=1}^{k} \frac{c_{a}}{c_{0}} \mathbb{E}\left(C_{a} \tilde{Q}\right)_{i i^{\prime}}-(p z)^{-1} \sum_{a=1}^{k} \mathbb{E}\left(C_{a} \tilde{Q} W \mathcal{D}\left(j_{a}\right) W^{\top} \tilde{Q}\right)_{i i^{\prime}} \\
& -(p z)^{-1} \sum_{a=1}^{k} \mathbb{E}\left(C_{a} \tilde{Q}\right)_{i i^{\prime}} \operatorname{tr}\left(\mathcal{D}\left(j_{a}\right) W^{\top} \tilde{Q} W\right)
\end{aligned}
$$

Using $W^{\top} \tilde{Q} W=W^{\top} W Q=z Q+I_{n}$, we then obtain

$$
\begin{aligned}
\mathbb{E} \tilde{Q}_{i i^{\prime}}= & -\delta_{i i^{\prime}} z^{-1}+z^{-1} \sum_{a=1}^{k} \frac{c_{a}}{c_{0}} \mathbb{E}\left(C_{a} \tilde{Q}\right)_{i i^{\prime}}-(p z)^{-1} \sum_{a=1}^{k} \mathbb{E}\left(C_{a} \tilde{Q} W \mathcal{D}\left(j_{a}\right) W^{\top} \tilde{Q}\right)_{i i^{\prime}} \\
& -\sum_{a=1}^{k} \mathbb{E}\left(C_{a} \tilde{Q}\right)_{i i^{\prime}} \frac{1}{p} \operatorname{tr}\left(\mathcal{D}\left(j_{a}\right) Q\right)-z^{-1} \sum_{a=1}^{k} \frac{c_{a}}{c_{0}} \mathbb{E}\left(C_{a} \tilde{Q}\right)_{i i^{\prime}} \\
= & -\delta_{i i^{\prime}} z^{-1}-(p z)^{-1} \sum_{a=1}^{k} \mathbb{E}\left(C_{a} \tilde{Q} W \mathcal{D}\left(j_{a}\right) W^{\top} \tilde{Q}\right)_{i i^{\prime}}-\sum_{a=1}^{k} \mathbb{E}\left(C_{a} \tilde{Q}\right)_{i i^{\prime}} \frac{1}{p} \operatorname{tr}\left(\mathcal{D}\left(j_{a}\right) Q\right)
\end{aligned}
$$

Thus for

$$
M_{2}:=\frac{1}{z} \sum_{a=1}^{k} \mathbb{E}\left(C_{a} \tilde{Q} W \mathcal{D}\left(j_{a}\right) W^{\top} \tilde{Q}\right)
$$


we have

$$
-z \mathbb{E} \tilde{Q}=I+z \sum_{a=1}^{k} \mathbb{E} \frac{1}{p} \operatorname{tr}\left(\mathcal{D}_{a} Q\right) C_{a} \tilde{Q}+z p^{-1} M_{2},
$$

i.e.

$$
\mathbb{E}\left(I_{p}+\sum_{a=1}^{p} \frac{1}{p} \operatorname{tr}\left(Q \mathcal{D}_{a}\right) C_{a}\right) \tilde{Q}=-z^{-1}\left(I_{p}-p^{-1} M_{2}\right),
$$

so that, for

$$
K:=\mathbb{E} \frac{1}{p} \operatorname{tr}\left(\mathcal{D}_{a} Q\right) \tilde{Q}-\mathbb{E} \frac{1}{p} \operatorname{tr}\left(\mathcal{D}_{a} Q\right) \mathbb{E} \tilde{Q}
$$

we have

$$
\begin{aligned}
\mathbb{E} \tilde{Q}= & \left(\mathbb{E}\left(I_{p}+\sum_{a=1}^{p} \frac{1}{p} \operatorname{tr}\left(Q \mathcal{D}_{a}\right) C_{a}\right)\right)^{-1} \mathbb{E}\left(I_{p}+\sum_{a=1}^{p} \frac{1}{p} \operatorname{tr}\left(Q \mathcal{D}_{a}\right) C_{a}\right) \mathbb{E} \tilde{Q} \\
= & \left(\mathbb{E}\left(I_{p}+\sum_{a=1}^{p} \frac{1}{p} \operatorname{tr}\left(Q \mathcal{D}_{a}\right) C_{a}\right)\right)^{-1}\left(\mathbb{E}\left(I_{p}+\sum_{a=1}^{p} \frac{1}{p} \operatorname{tr}\left(Q \mathcal{D}_{a}\right) C_{a}\right) \tilde{Q}-K\right) \\
= & -\left(\mathbb{E}\left(z I_{p}+\sum_{a=1}^{p} \frac{z}{p} \operatorname{tr}\left(Q \mathcal{D}_{a}\right) C_{a}\right)\right)^{-1}+p^{-1}\left(\mathbb{E}\left(z I_{p}+\sum_{a=1}^{p} \frac{z}{p} \operatorname{tr}\left(Q \mathcal{D}_{a}\right) C_{a}\right)\right)^{-1} M_{2} \\
& -\left(\mathbb{E}\left(z I_{p}+\sum_{a=1}^{p} \frac{z}{p} \operatorname{tr}\left(Q \mathcal{D}_{a}\right) C_{a}\right)\right)^{-1} K .
\end{aligned}
$$

Note that for any $a \in\{1, \ldots, k\}$, by Lemma B.1,

$$
\|K\|=\left\|\mathbb{E}\left[\left(\frac{1}{p} \operatorname{tr}\left(\mathcal{D}_{a} Q\right)-\mathbb{E} \frac{1}{p} \operatorname{tr}\left(\mathcal{D}_{a} Q\right)\right) \tilde{Q}\right]\right\| \leq \frac{1}{|\Im z|} \sqrt{\operatorname{Var} \frac{1}{p} \operatorname{tr}\left(\mathcal{D}_{a} Q\right)}=O\left(p^{-1}\right) .
$$

Besides, we also have $\left\|M_{2}\right\|=O(1)$ and

$$
\left\|\left(\mathbb{E}\left(z I_{p}+\sum_{a=1}^{p} z p^{-1} \operatorname{tr}\left(Q \mathcal{D}_{a}\right) C_{a}\right)\right)^{-1}\right\| \leq|\Im z|^{-1},
$$

hence

$$
\mathbb{E} \tilde{Q}=-z^{-1}\left(I_{p}+\sum_{a=1}^{k} \mathbb{E} \frac{1}{p} \operatorname{tr}\left(\mathcal{D}\left(j_{a}\right) Q\right) C_{a}\right)^{-1}+O_{\|\cdot\|}\left(p^{-1}\right) .
$$

This proves (4.4).

\subsubsection{Consequences of the loop equations: Proof of Lemma 4.2}

We have proved

$$
\begin{aligned}
& \mathbb{E} Q=-z^{-1}\left(I_{n}+\mathbb{E} D\right)^{-1}+O_{\|\cdot\|}\left(p^{-1}\right) \\
& \mathbb{E} \tilde{Q}=-z^{-1}\left(I_{p}+\sum_{a=1}^{k} \mathbb{E} \frac{1}{p} \operatorname{tr}\left(\mathcal{D}\left(j_{a}\right) Q\right) C_{a}\right)^{-1}+O_{\|\cdot\|}\left(p^{-1}\right)
\end{aligned}
$$


for $D=\sum_{a=1}^{k} \frac{1}{p} \operatorname{tr}\left(\tilde{Q} C_{a}\right) \mathcal{D}_{a}$. This is precisely the content of Lemma 4.2. It also implies that, for the key complex quantities

$$
G_{a}=G_{a}(z):=\mathbb{E} \frac{1}{c_{0} n_{a}} \operatorname{tr}\left(\mathcal{D}\left(j_{a}\right) Q\right)=\mathbb{E} \frac{1}{p c_{a}} \operatorname{tr}\left(\mathcal{D}\left(j_{a}\right) Q\right), \quad \tilde{G}_{a}(z):=\mathbb{E} \frac{1}{p} \operatorname{tr}\left(\tilde{Q} C_{a}\right),
$$

we have

$$
\begin{aligned}
c_{0} G_{a} & =-z^{-1}\left(1+\tilde{G}_{a}\right)^{-1}+O\left(p^{-1}\right) \\
\tilde{G}_{a} & =-z^{-1} \frac{1}{p} \operatorname{tr} C_{a}\left(I+\sum_{b=1}^{k} c_{b} G_{b} C_{b}\right)^{-1}+O\left(p^{-1}\right),
\end{aligned}
$$

which implies that

$$
c_{0} G_{a}=\frac{1}{-z+\frac{1}{p} \operatorname{tr} C_{a}\left(I+\sum_{b=1}^{k} c_{b} G_{b} C_{b}\right)^{-1}+O\left(p^{-1}\right)} .
$$

\subsection{Proof of Proposition 1.5}

It follows from Lemma 2.1 and from (4.13) that, for $G_{a}$ as defined in (4.11) and for $z \in \mathbb{C} \backslash \mathbb{R}$,

$$
\left|g_{a}(z)-G_{a}(z)\right|=O\left(p^{-1}\right)
$$

From Lemma 4.2, we deduce that, with the notations of Proposition 1.5,

$$
\mathbb{E} Q_{z}-\bar{Q}_{z}=O_{\|\cdot\|}\left(p^{-1}\right), \quad \mathbb{E} \tilde{Q}_{z}-\overline{\tilde{Q}}_{z}=O_{\|\cdot\|}\left(p^{-1}\right) .
$$

By the concentration Lemma B.1, we immediately deduce Proposition 1.5 as long as $z$ stays at a macroscopic distance from $\mathbb{R}$.

To extend the result to all $z$ 's taken at a macroscopic distance from $\mathcal{S} \cup\{0\}$, we shall prove next that the spectrum of $W^{\top} W$ remains almost surely away from $\mathcal{S} \cup\{0\}$ (thus proving in passing (1.3) of Thm. 1.1). Let $\mathcal{I}$ be a closed interval of $\mathbb{R}$ at a macroscopic distance from $\mathcal{S} \cup\{0\}$. There is $\varepsilon>0$ such that the distance from $\mathcal{I}$ to $\mathcal{S} \cup\{0\}$ is at least $2 \varepsilon$. Let $\eta>0$ such that $\left(2 \varepsilon^{2}+\eta^{2}\right)^{1 / 2}-\eta=\varepsilon$. We have

$$
\sup _{x \in \mathcal{I}}\left\|\bar{Q}_{x+\mathrm{i} \eta}\right\| \leq\left(4 \varepsilon^{2}+\eta^{2}\right)^{-1 / 2} .
$$

We deduce that for $p$ large enough,

$$
\sup _{x \in \mathcal{I}}\left\|\mathbb{E} Q_{x+\mathrm{i} \eta}\right\| \leq\left(3 \varepsilon^{2}+\eta^{2}\right)^{-1 / 2}
$$

Hence by measure concentration (using the arguments of the proof of [20], Cor. 6), with probability tending to one,

$$
\sup _{x \in \mathcal{I}} \sup _{\lambda \in \operatorname{Spec}\left(W^{\top} W\right)}|\lambda-(x+\mathrm{i} \eta)|^{-1}=\sup _{x \in \mathcal{I}}\left\|Q_{x+i \eta}\right\| \leq\left(2 \varepsilon^{2}+\eta^{2}\right)^{-1 / 2},
$$

i.e. that

$$
\inf _{x \in \mathcal{I}} \inf _{\lambda \in \operatorname{Spec}\left(W^{\top} W\right)}|\lambda-(x+\mathrm{i} \eta)| \geq\left(2 \varepsilon^{2}+\eta^{2}\right)^{1 / 2}
$$

which implies finally that

$$
\inf _{x \in \mathcal{I}} \inf _{\lambda \in \operatorname{Spec}\left(W^{\top} W\right)}|\lambda-x| \geq\left(2 \varepsilon^{2}+\eta^{2}\right)^{1 / 2}-\eta=\varepsilon .
$$

This being true for any such interval $\mathcal{I}$, by the union bound and Lemma 4.1, we have the sought for result. 


\subsection{Proof of Proposition 1.6}

Our first interest is on $Q_{z_{1}} \mathcal{D}_{a} Q_{z_{2}}$. By the resolvent identity $W^{\top} W Q_{z}-z Q_{z}=I_{p}$ applied to either of the two matrices $Q_{z_{1}}$ or $Q_{z_{2}}$, along with Stein's lemma and the results from Proposition 1.5, we then get (technical details, similar to previous derivations, are omitted)

$$
\begin{aligned}
\mathbb{E}\left[Q_{z_{1}} \mathcal{D}_{a} Q_{z_{2}}\right]_{i j}= & -\frac{1}{z_{1}}\left[\mathcal{D}_{a} \bar{Q}_{z_{2}}\right]_{i j}-\sum_{b=1}^{k} \tilde{g}_{b}\left(z_{1}\right)\left[\mathcal{D}_{b} \bar{Q}_{z_{1}} \mathcal{D}_{a} \bar{Q}_{z_{2}}\right]_{i j} \\
& -\frac{1}{z_{1}} \sum_{b=1}^{k} r_{a b}\left(z_{1}, z_{2}\right) \mathbb{E}\left[\mathcal{D}_{b} \bar{Q}_{z_{2}}\right]_{i j}+O\left(p^{-1}\right),
\end{aligned}
$$

where we defined

$$
r_{a b}\left(z_{1}, z_{2}\right):=\mathbb{E}\left[p^{-1} \operatorname{tr}\left(\mathcal{D}_{a} W^{\top} \tilde{Q}_{z_{1}} C_{b} \tilde{Q}_{z_{2}} W\right)\right]
$$

Similarly, we find

$$
\mathbb{E}\left[\tilde{Q}_{z_{1}} W \mathcal{D}_{a} W^{\top} \tilde{Q}_{z_{2}}\right]_{i j}=z_{1} z_{2} g_{a}\left(z_{1}\right) g_{a}\left(z_{2}\right) c_{a} c_{0} \mathbb{E}\left[\tilde{Q}_{z_{1}} C_{a} \tilde{Q}_{z_{2}}\right]_{i j}+O\left(p^{-1}\right)
$$

which introduces the term $\mathbb{E}\left[\tilde{Q}_{z_{1}} C_{a} \tilde{Q}_{z_{2}}\right]_{i j}$. This term is also similarly treated and gives

$$
\mathbb{E}\left[\left[\tilde{Q}_{z_{1}} C_{a} \tilde{Q}_{z_{2}}\right]_{i j}\right]=\left[\overline{\tilde{Q}}_{z_{1}} C_{a} \overline{\tilde{Q}}_{z_{2}}\right]_{i j}+\sum_{b=1}^{k} r_{b a}\left(z_{1}, z_{2}\right)\left[\overline{\tilde{Q}}_{z_{1}} C_{b} \overline{\tilde{Q}}_{z_{2}}\right]_{i j}+O\left(p^{-1}\right) .
$$

To wrap up the various results, we need to identify precisely $r_{a b}\left(z_{1}, z_{2}\right)$. To this end, from (4.16), we find

$$
\begin{aligned}
r_{a b}\left(z_{1}, z_{2}\right) & =z_{1} z_{2} g_{a}\left(z_{1}\right) g_{a}\left(z_{2}\right) c_{a} c_{0} \mathbb{E}\left[\frac{1}{p} \operatorname{tr}\left(C_{a} \tilde{Q}_{z_{1}} C_{b} \tilde{Q}_{z_{2}}\right)\right]+O\left(p^{-1}\right) \\
& =z_{1} z_{2} g_{a}\left(z_{1}\right) g_{a}\left(z_{2}\right) c_{a} c_{0} \frac{1}{p} \operatorname{tr}\left(C_{a} \overline{\tilde{Q}}_{z_{1}} C_{b} \overline{\tilde{Q}}_{z_{2}}\right) \\
& +z_{1} z_{2} g_{a}\left(z_{1}\right) g_{a}\left(z_{2}\right) c_{a} c_{0} \sum_{d=1}^{k} r_{d a}\left(z_{2}, z_{1}\right) \frac{1}{p} \operatorname{tr}\left(C_{d} \overline{\tilde{Q}}_{z_{1}} C_{b} \overline{\tilde{Q}}_{z_{2}}\right)+O\left(p^{-1}\right) .
\end{aligned}
$$

From the definition of $r_{a b}\left(z_{1} z_{2}\right)$, it is clear that $r_{d a}\left(z_{2}, z_{1}\right) c_{a} g_{a}\left(z_{1}\right) g_{a}\left(z_{2}\right)=r_{a d}\left(z_{1}, z_{2}\right) c_{d} g_{d}\left(z_{1}\right) g_{d}\left(z_{2}\right)$. Thus, the above formula can be rewritten

$$
\begin{gathered}
r_{a b}\left(z_{1}, z_{2}\right)-z_{1} z_{2} c_{0} \sum_{d=1}^{k} r_{a d}\left(z_{1}, z_{2}\right) c_{d} g_{d}\left(z_{1}\right) g_{d}\left(z_{2}\right) \frac{1}{p} \operatorname{tr}\left(C_{d} \overline{\tilde{Q}}_{z_{1}} C_{b} \overline{\tilde{Q}}_{z_{2}}\right)=z_{1} z_{2} c_{0} c_{a} g_{a}\left(z_{1}\right) g_{a}\left(z_{2}\right) \\
\times \frac{1}{p} \operatorname{tr}\left(C_{a} \overline{\tilde{Q}}_{z_{1}} C_{b} \overline{\tilde{Q}}_{z_{2}}\right)+O\left(p^{-1}\right) .
\end{gathered}
$$

This can be further rewritten under a matrix form which, after basic manipulations, leads finally to

$$
r_{a b}\left(z_{1}, z_{2}\right)=\left[R\left(z_{1}, z_{2}\right)\right]_{a b}+O\left(p^{-1}\right)
$$

with $R\left(z_{1}, z_{2}\right)$ defined in the statement of the lemma. The fact that $\left(I_{k}-\Omega\left(z_{1}, z_{2}\right)\right)^{-1}$ in the expression of $R\left(z_{1}, z_{2}\right)$ is well-defined as an inverse matrix is a consequence of $\rho\left(\Omega\left(z_{1}, z_{2}\right)\right)<1$ by Lemma 2.1 (ii) with $\varepsilon=\eta=0$, for every $z_{1}, z_{2} \in \mathbb{C} \backslash \mathbb{R}$. The proof of the proposition is then completed by applying the concentration result from Lemma B.1. 


\section{Proof of Theorem 1.1}

The proof of (1.2) follows directly from Proposition 1.5. As for the proof of (1.3), it was already obtained in the proof of Proposition 1.5. Let us then prove the remaining second part of Theorem 1.1.

For $z \in \mathbb{C}^{+}$, denote $g(z)=\left(g_{1}(z), \ldots, g_{k}(z)\right)^{\top}$ with $g_{1}(z), \ldots, g_{k}(z)$ defined by Proposition 1.3. By Lemma 2.1 (i) with $\varepsilon=0, \eta=0$, we have, for any $z_{1}, z_{2} \in \mathbb{C} \backslash \mathbb{R}$,

$$
\left(I_{k}-\Omega\left(z_{1}, z_{2}\right)\right)\left(g\left(z_{1}\right)-g\left(z_{2}\right)\right)=\left(z_{1}-z_{2}\right) c_{0} g\left(z_{1}\right) \odot g\left(z_{2}\right)
$$

where

$$
\Omega\left(z_{1}, z_{2}\right):=c_{0} z_{1} z_{2}\left\{c_{b} g_{a}\left(z_{1}\right) g_{a}\left(z_{2}\right) \frac{1}{p} \operatorname{tr} C_{a} \overline{\tilde{Q}}_{z_{1}} C_{b} \overline{\tilde{Q}}_{z_{2}}\right\}_{a, b=1}^{k}
$$

However, it is not convenient for our present investigation to work with $\Omega\left(z_{1}, z_{2}\right)$ which does not exhibit enough symmetry. We shall then proceed next by left-multiplying both sides of $(5.1)$ by $\operatorname{diag}(c)^{\frac{1}{2}} \operatorname{diag}\left(g\left(z_{1}\right) \odot\right.$ $\left.g\left(z_{2}\right)\right)^{-\frac{1}{2}}$, where the complex square root is defined thanks to the natural definition of the argument on $\mathbb{C} \backslash \mathbb{R}_{+}$ (resp. $\mathbb{C} \backslash \mathbb{R}_{-}$) if $\Im z_{1} \Im z_{2}>0$ (resp. if $\Im z_{1} \Im z_{2}<0$ ). This entails

$$
\left(I_{k}-\Upsilon\left(z_{1}, z_{2}\right)\right)\left\{\sqrt{c_{a}} \frac{g_{a}\left(z_{1}\right)-g_{a}\left(z_{2}\right)}{\sqrt{g_{a}\left(z_{1}\right) g_{a}\left(z_{2}\right)}}\right\}_{a=1}^{k}=\left(z_{1}-z_{2}\right) c_{0}\left\{\sqrt{c_{a} g_{a}\left(z_{1}\right) g_{a}\left(z_{2}\right)}\right\}_{a=1}^{k}
$$

where we defined

$$
\Upsilon\left(z_{1}, z_{2}\right)_{a b}:=c_{0} z_{1} z_{2} \sqrt{c_{a} c_{b}} \sqrt{g_{a}\left(z_{1}\right) g_{b}\left(z_{1}\right) g_{a}\left(z_{2}\right) g_{b}\left(z_{2}\right)} \frac{1}{p} \operatorname{tr} C_{a} \overline{\tilde{Q}}_{z_{1}} C_{b} \overline{\tilde{Q}}_{z_{2}} .
$$

The matrix $\Upsilon\left(z_{1}, z_{2}\right)$ is "more" symmetrical than $\Omega\left(z_{1}, z_{2}\right)$ but satisfies only $\Upsilon\left(z_{1}, z_{2}\right)_{a b}=\Upsilon\left(z_{2}, z_{1}\right)_{b a}$, which shall not be good enough in what follows. To symmetrize this expression further, observe that, exchanging $z_{1}$ and $z_{2}$, we also get

$$
\left(I_{k}-\Upsilon\left(z_{2}, z_{1}\right)\right)\left\{\sqrt{c_{a}} \frac{g_{a}\left(z_{1}\right)-g_{a}\left(z_{2}\right)}{\sqrt{g_{a}\left(z_{1}\right) g_{a}\left(z_{2}\right)}}\right\}_{a=1}^{k}=\left(z_{1}-z_{2}\right) c_{0}\left\{\sqrt{c_{a} g_{a}\left(z_{1}\right) g_{a}\left(z_{2}\right)}\right\}_{a=1}^{k}
$$

so that, summing up the two equations leads to

$$
\left(I_{k}-\Xi\left(z_{1}, z_{2}\right)\right)\left\{\sqrt{c_{a}} \frac{g_{a}\left(z_{1}\right)-g_{a}\left(z_{2}\right)}{\sqrt{g_{a}\left(z_{1}\right) g_{a}\left(z_{2}\right)}}\right\}_{a=1}^{k}=\left(z_{1}-z_{2}\right) c_{0}\left\{\sqrt{c_{a} g_{a}\left(z_{1}\right) g_{a}\left(z_{2}\right)}\right\}_{a=1}^{k}
$$

where

$$
\Xi\left(z_{1}, z_{2}\right):=\frac{1}{2}\left(\Upsilon\left(z_{1}, z_{2}\right)+\Upsilon\left(z_{2}, z_{1}\right)\right)
$$

In particular,

$$
\left(I_{k}-\Xi\left(z, z^{*}\right)\right)\left\{\sqrt{c_{a}} \frac{\Im\left(g_{a}(z)\right)}{\left|g_{a}(z)\right|}\right\}_{a=1}^{k}=\Im(z) c_{0}\left\{\sqrt{c_{a}}\left|g_{a}(z)\right|\right\}_{a=1}^{k}
$$

where $\Xi\left(z, z^{*}\right)$ is real positive and symmetric. Hence, by Lemma C.1, we may take $x$ with positive entries a left eigenvector of $\Xi\left(z, z^{*}\right)$ with eigenvalue $\rho\left(\Xi\left(z, z^{*}\right)\right)$. Multiplying by $x$ on the left, we get $\rho\left(\Xi\left(z, z^{*}\right)\right)<1$. Thus, $\Xi\left(z, z^{*}\right)$ is invertible for every $z \in \mathbb{C}^{+}$and we thus have

$$
\left\{\sqrt{c_{a}} \frac{\Im\left(g_{a}(z)\right)}{\left|g_{a}(z)\right|}\right\}_{a=1}^{k}=\Im(z) c_{0}\left(I_{k}-\Xi\left(z, z^{*}\right)\right)^{-1}\left\{\sqrt{c_{a}}\left|g_{a}(z)\right|\right\}_{a=1}^{k} .
$$


Using now the fact that

$$
\begin{aligned}
|\operatorname{tr}(A B+C D)|^{2} & =\left|\operatorname{tr}\left([A C]\left[\begin{array}{l}
B \\
D
\end{array}\right]\right)\right|^{2} \\
& \leq \operatorname{tr}\left([A C]\left[\begin{array}{l}
A^{*} \\
C^{*}
\end{array}\right]\right) \operatorname{tr}\left(\left[B^{*} D^{*}\right]\left[\begin{array}{l}
B \\
D
\end{array}\right]\right) \\
& =\operatorname{tr}\left(A A^{*}+C C^{*}\right) \operatorname{tr}\left(B B^{*}+D D^{*}\right)
\end{aligned}
$$

applied to $A=C_{a}^{\frac{1}{2}} \overline{\tilde{Q}}_{z_{1}} C_{b}^{\frac{1}{2}}, B=C_{b}^{\frac{1}{2}} \overline{\tilde{Q}}_{z_{2}} C_{a}^{\frac{1}{2}}, C=C_{b}^{\frac{1}{2}} \overline{\tilde{Q}}_{z_{1}} C_{a}^{\frac{1}{2}}$, and $D=C_{a}^{\frac{1}{2}} \overline{\tilde{Q}}_{z_{2}} C_{b}^{\frac{1}{2}}$, we find that

$$
\left|\Xi\left(z_{1}, z_{2}\right)_{a b}\right|^{2} \leq \Xi\left(z_{1}, z_{1}^{*}\right)_{a b} \Xi\left(z_{2}, z_{2}^{*}\right)_{a b}
$$

and thus, from Lemma C.3, we get that $\rho\left(\Xi\left(z_{1}, z_{2}\right)\right)<1$ for each $z_{1}, z_{2} \in \mathbb{C}^{+}$. But since $\Xi\left(z, z^{*}\right)_{a b} \leq$ $\left\|\Xi\left(z, z^{*}\right)\right\|=\rho\left(\Xi\left(z, z^{*}\right)\right)<1$ for symmetric matrices, we have in addition $\left|\Xi\left(z_{1}, z_{2}\right)_{a b}\right|^{2} \leq 1$ for each $a, b$ so that, by e.g., $A^{-1}=\frac{\operatorname{adj}(A)}{\operatorname{det} A}$, we finally get

$$
\left\|\left(I_{k}-\Xi\left(z_{1}, z_{2}\right)\right)^{-1}\right\| \leq \frac{K}{\left|1-\rho\left(\Xi\left(z_{1}, z_{2}\right)\right)\right|^{k}}
$$

for some constant $K>0$, and in particular

$$
\left\{\sqrt{c_{a}} \frac{g_{a}\left(z_{1}\right)-g_{a}\left(z_{2}\right)}{\sqrt{g_{a}\left(z_{1}\right) g_{a}\left(z_{2}\right)}}\right\}_{a=1}^{k}=\left(z_{1}-z_{2}\right) c_{0}\left(I_{k}-\Xi\left(z_{1}, z_{2}\right)\right)^{-1}\left\{\sqrt{c_{a} g_{a}\left(z_{1}\right) g_{a}\left(z_{2}\right)}\right\}_{a=1}^{k} .
$$

With this identity at hand, we shall show that $g(z)$ admits a limit as $z \in \mathbb{C}^{+} \rightarrow x \in \mathbb{R}^{*}$. This will be sufficient by ([24], Thms. 2.1-2.2) to ensure that $\mu$ admits a continuous density on $\mathbb{R}^{*}$.

Recall first the notation

$$
\tilde{g}_{a}(z):=\frac{1}{p} \operatorname{tr} C_{a} \overline{\tilde{Q}}_{z}, \quad \overline{\tilde{Q}}_{z}=-z^{-1}\left(I_{p}+\sum_{b=1}^{k} c_{b} g_{b}(z) C_{b}\right)^{-1}
$$

(so that $c_{0} g_{a}(z)=-z^{-1}\left(1+\tilde{g}_{a}(z)\right)^{-1}$ ). Then we have the following first result.

Lemma 5.1. For any $\varepsilon>0, g(z)$ is bounded on $\left\{z \in \mathbb{C}^{+} ;|z|>\varepsilon\right\}$.

Proof. Note first that, by the inequality $\left|\operatorname{tr} A B^{*}\right|^{2} \leq \operatorname{tr} A A^{*} \operatorname{tr} B B^{*}$ with $B=I$,

$$
\left|\tilde{g}_{a}(z)\right|^{2}=\left|\frac{1}{p} \operatorname{tr} C_{a}^{\frac{1}{2}} \overline{\tilde{Q}}_{z} C_{a}^{\frac{1}{2}}\right|^{2} \leq \frac{1}{p} \operatorname{tr} C_{a} \overline{\tilde{Q}}_{z} C_{a} \overline{\tilde{Q}}_{z^{*}}
$$

so that

$$
c_{0} c_{a}\left|z g_{a}(z) \tilde{g}_{a}(z)\right|^{2} \leq c_{0} c_{a}\left|z g_{a}(z)\right|^{2} \frac{1}{p} \operatorname{tr} C_{a} \overline{\tilde{Q}}_{z} C_{a} \overline{\tilde{Q}}_{z^{*}}=\Xi\left(z, z^{*}\right)_{a a} .
$$

Since $\Xi\left(z, z^{*}\right)_{a a} \leq \rho\left(\Xi\left(z, z^{*}\right)\right)<1$, we thus get that for each $z \in \mathbb{C}^{+}, c_{0} c_{a}\left|z g_{a}(z) \tilde{g}_{a}(z)\right|^{2}<1$. Hence, if $\left|g_{a}\left(z_{n}\right)\right| \rightarrow \infty$ on some sequence with $\left|z_{n}\right|>\varepsilon$, this implies that $\left|\tilde{g}_{a}\left(z_{n}\right)\right| \rightarrow 0$. But by definition, $\left|g_{a}\left(z_{n}\right)\right|=$ $\left|z_{n} c_{0}\left(1+\tilde{g}_{a}\left(z_{n}\right)\right)\right|^{-1}$, which is thus bounded, contradicting the assumption. We conclude that $g_{a}(z)$ must remain bounded on $\left\{z \in \mathbb{C}^{+} ;|z|>\varepsilon\right\}$.

Lemma 5.2. Under the additional assumptions of Theorem 1.1, for any $x_{0} \in \mathbb{R}^{*}, g(z)$ admits a finite limit as $z \in \mathbb{C}^{+}$tends to $x_{0}$. 
Proof. If not, by the previous lemma, one can find two sequences $z_{n}^{1}, z_{n}^{2} \in \mathbb{C}^{+}$tending to $x_{0} \in \mathbb{R}^{*}$ such that $g\left(z_{n}^{1}\right) \rightarrow g^{1}$ and $g\left(z_{2}^{n}\right) \rightarrow g^{2}, g^{1} \neq g^{2}$. From (5.4),

$$
\left\{\sqrt{c_{a}} \frac{g_{a}\left(z_{n}^{1}\right)-g\left(z_{n}^{2}\right)}{\sqrt{g_{a}\left(z_{1}^{n}\right) g_{a}\left(z_{n}^{2}\right)}}\right\}_{a=1}^{k}=\left(z_{n}^{1}-z_{n}^{2}\right) c_{0}\left(I_{k}-\Xi\left(z_{n}^{1}, z_{n}^{2}\right)\right)^{-1}\left\{\sqrt{c_{a} g_{a}\left(z_{n}^{1}\right) g_{a}\left(z_{n}^{2}\right)}\right\}_{a=1}^{k} .
$$

Since $z_{n}^{1}, z_{n}^{2} \rightarrow x_{0}, z_{n}^{1}-z_{n}^{2} \rightarrow 0$. Also, since $g\left(z_{n}^{1}\right), g\left(z_{n}^{2}\right)$ are bounded by the previous lemma, we get that $\left(z_{n}^{1}-z_{n}^{2}\right) c_{0} \sqrt{c_{a} g_{a}\left(z_{n}^{1}\right) g_{a}\left(z_{n}^{2}\right)} \rightarrow 0$. It thus remains to show that $\left(I_{k}-\Xi\left(z_{n}^{1}, z_{n}^{2}\right)\right)^{-1}$ has uniformly bounded spectral norm, which, by (5.3), is equivalent to showing that

$$
\limsup _{n} \rho\left(\Xi\left(z_{n}^{1}, z_{n}^{2}\right)\right)<1 .
$$

Recall first that we obtained, from Lemma C.3 and

$$
\left|\Xi\left(z_{n}^{1}, z_{n}^{2}\right)_{a b}\right|^{2} \leq \Xi\left(z_{n}^{1},\left(z_{n}^{1}\right)^{*}\right)_{a b} \Xi\left(z_{n}^{2},\left(z_{n}^{2}\right)^{*}\right)_{a b},
$$

that $\rho\left(\Xi\left(z_{n}^{1}, z_{n}^{2}\right)\right) \leq \sqrt{\rho\left(\Xi\left(z_{n}^{1},\left(z_{n}^{1}\right)^{*}\right)\right) \rho\left(\Xi\left(z_{n}^{2},\left(z_{n}^{2}\right)^{*}\right)\right)}$. Since $\rho\left(\Xi\left(z, z^{*}\right)\right)<1$ for each $z \in \mathbb{C}^{+}$, in the limit, this only ensures that $\lim _{\sup } \rho\left(\Xi\left(z_{n}^{1}, z_{n}^{2}\right)\right) \leq 1$. We may thus show that the inequality $\left|\Xi\left(z_{n}^{1}, z_{n}^{2}\right)_{a a}\right|^{2} \leq$ $\Xi\left(z_{n}^{1},\left(z_{n}^{1}\right)^{*}\right)_{a a} \Xi\left(z_{n}^{2},\left(z_{n}^{2}\right)^{*}\right)_{a a}$ is strict, uniformly in $n$, for each $a$. To this end, we shall use the second part of Lemma C.3.

Letting $U_{n}^{1}:=C_{a}^{\frac{1}{2}} \overline{\tilde{Q}}_{z_{n}^{1}} C_{a}^{\frac{1}{2}}$ and $U_{n}^{2}:=C_{a}^{\frac{1}{2}} \overline{\tilde{Q}}_{\left(z_{n}^{2}\right)^{*}} C_{a}^{\frac{1}{2}}$, we wish to show that, uniformly on $\lambda \in \mathbb{C}$,

$$
\liminf _{n} \operatorname{tr}\left(U_{n}^{1}-\lambda U_{n}^{2}\right)\left(U_{n}^{1}-\lambda U_{n}^{2}\right)^{*}>0 .
$$

For this, note that, for each $\lambda \in \mathbb{C}$,

$$
\operatorname{tr}\left(U_{n}^{1}-\lambda U_{n}^{2}\right)\left(U_{n}^{1}-\lambda U_{n}^{2}\right)^{*}=\operatorname{tr} C_{a}^{\frac{1}{2}} \overline{\tilde{Q}}_{z_{n}^{1}} \Delta_{n}^{Q} \overline{\tilde{Q}}_{z_{n}^{2}}^{*} C_{a} \overline{\tilde{Q}}_{z_{n}^{2}}\left(\Delta_{n}^{Q}\right)^{*} \overline{\tilde{Q}}_{z_{n}^{1}}^{*} C_{a}^{\frac{1}{2}}
$$

with

$$
\Delta_{n}^{Q}:=\left(\lambda z_{n}^{1}-\left(z_{n}^{2}\right)^{*}\right) I_{p}+\sum_{i=1}^{k} c_{i}\left(\lambda z_{n}^{1} g_{i}\left(z_{n}^{1}\right)-\left(z_{n}^{2}\right)^{*} g_{i}\left(\left(z_{n}^{2}\right)^{*}\right)\right) C_{i} .
$$

From the fact that $\operatorname{tr} A B A^{*} \geq \lambda_{\min }(B) \operatorname{tr} A A^{*}$ when $B$ is nonnegative definite, we then get

$$
\operatorname{tr}\left(U_{n}^{1}-\lambda U_{n}^{2}\right)\left(U_{n}^{1}-\lambda U_{n}^{2}\right)^{*} \geq \lambda_{\min }\left(\overline{\tilde{Q}}_{z_{n}^{2}}^{*} C_{a} \overline{\tilde{Q}}_{z_{n}^{2}}\right) \lambda_{\min }\left(\overline{\tilde{Q}}_{z_{n}^{1}}^{*} C_{a} \overline{\tilde{Q}}_{z_{n}^{1}}\right) \operatorname{tr} \Delta_{n}^{Q}\left(\Delta_{n}^{Q}\right)^{*} .
$$

Exploiting the invertibility of $C_{a}$ along with the fact that $\left\|\overline{\tilde{Q}}_{z}^{-1}\right\|$ is bounded uniformly on $z \in \mathbb{C}^{+}$away from zero (by the previous lemma), we then get that

$$
\liminf _{n} \lambda_{\min }\left(\overline{\tilde{Q}}_{z_{n}^{2}}^{*} C_{a} \overline{\tilde{Q}}_{z_{n}^{2}}\right) \lambda_{\min }\left(\overline{\tilde{Q}}_{z_{n}^{1}}^{*} C_{a} \overline{\tilde{Q}}_{z_{n}^{1}}\right)>0 .
$$

By the boundedness of $g$ away from zero, we also have

$$
\lim _{n} \operatorname{tr} \Delta_{n}^{Q}\left(\Delta_{n}^{Q}\right)^{*}=\operatorname{tr} \Delta^{Q}\left(\Delta^{Q}\right)^{*}
$$

with

$$
\Delta^{Q}=x_{0}\left[(\lambda-1) I_{p}+\sum_{i=1}^{k} c_{i}\left(\lambda g_{i}^{1}-\left(g_{i}^{2}\right)^{*}\right) C_{i}\right]
$$


By linear independence of the matrices $C_{1}, \ldots, C_{k}, I_{p}$, the quantity above cannot be zero unless $\lambda=1$ and $g_{i}^{1}=\left(g_{i}^{2}\right)^{*}$ for each $i$. But $\Im\left(g_{i}^{1}\right), \Im\left(g_{i}^{2}\right) \geq 0$ so that this implies $g_{i}^{1}=g_{i}^{2} \in \mathbb{R}$ for each $i$. But this is forbidden by assumption, and thus

$$
\inf _{\lambda \in \mathbb{C}} \liminf _{n} \operatorname{tr}\left(U_{n}^{1}-\lambda U_{n}^{2}\right)\left(U_{n}^{1}-\lambda U_{n}^{2}\right)^{*}>0
$$

This ensures (possibly over a converging subsequence, which exists for all quantities here are bounded) that

$$
\lim _{n} \operatorname{tr} U_{n}^{1}\left(U_{n}^{1}\right)^{*} \operatorname{tr} U_{n}^{2}\left(U_{n}^{2}\right)^{*}>\lim _{n}\left|\operatorname{tr} U_{n}^{1} U_{n}^{2}\right|^{2}
$$

Indeed, uniformly over $x \in \mathbb{R},(5.6)$ (applied to $\lambda=x / \sqrt{2}$ and $\mathrm{i} x / \sqrt{2}$ ) ensures that

$$
\begin{aligned}
& \lim _{n} \operatorname{tr} U_{n}^{1}\left(U_{n}^{1}\right)^{*}+\frac{1}{2} x^{2} \lim _{n} \operatorname{tr} U_{n}^{2}\left(U_{n}^{2}\right)^{*}>\sqrt{2} x \lim _{n} \Re\left(\operatorname{tr} U_{n}^{1}\left(U_{n}^{2}\right)^{*}\right) \\
& \lim _{n} \operatorname{tr} U_{n}^{1}\left(U_{n}^{1}\right)^{*}+\frac{1}{2} x^{2} \lim _{n} \operatorname{tr} U_{n}^{2}\left(U_{n}^{2}\right)^{*}>\sqrt{2} x \lim _{n} \Im\left(\operatorname{tr} U_{n}^{1}\left(U_{n}^{2}\right)^{*}\right) .
\end{aligned}
$$

Taking squares left and right on both equations, summing, and taking square-roots left and right on the result, this gives, uniformly on $x$,

$$
\lim _{n} \operatorname{tr} U_{n}^{1}\left(U_{n}^{1}\right)^{*}+\frac{1}{2} x^{2} \lim _{n} \operatorname{tr} U_{n}^{2}\left(U_{n}^{2}\right)^{*}-\sqrt{2} x \lim _{n}\left|\operatorname{tr} U_{n}^{1}\left(U_{n}^{2}\right)^{*}\right|>0,
$$

the left-hand side of which is a polynomial in $x$ with discriminant $2 \lim _{n}\left|\operatorname{tr} U_{n}^{1}\left(U_{n}^{2}\right)^{*}\right|^{2}-$ $2 \lim _{n} \operatorname{tr} U_{n}^{1}\left(U_{n}^{1}\right)^{*} \lim _{n} \operatorname{tr} U_{n}^{2}\left(U_{n}^{2}\right)^{*}$ which is positive.

All this finally proves, by Lemma C.3, that $\lim _{\sup _{n}} \rho\left(\Xi\left(z_{n}^{1}, z_{n}^{2}\right)\right)<1$ and therefore, recalling (5.3), the lefthand side of (5.5) converges to zero as $n \rightarrow \infty$, and so must the left-hand side. But since $g(z)$ is bounded away from zero, this implies that $g^{1}=g^{2}$, which goes against the assumption.

By ([24], Thms. 2.1 and 2.2), we then get that $\Im\left(g_{a}(z)\right)$ is continuous on $\mathbb{R}^{*}$ and $\nu_{a}$ has continuous derivative $f_{a}(x)=\frac{1}{\pi} \Im\left(g_{a}(x)\right)$. As $\mu=\sum_{a=1}^{k} c_{a} \nu_{a}$, the result follows.

\section{Appendix A. Multidimensional Stein formula}

Lemma A.1. Let $X=\left(X_{1}, \ldots, X_{d}\right)$ be a centered Gaussian vector and $f: \mathbb{R}^{d} \rightarrow \mathbb{R}$ be a $\mathcal{C}^{1}$ function with derivatives having at most polynomial growth. Then for all $i_{0}=1, \ldots, d$,

$$
\mathbb{E}\left[X_{i_{0}} f\left(X_{1}, \ldots, X_{d}\right)\right]=\sum_{k=1}^{d} \mathbb{E}\left[X_{i_{0}} X_{k}\right] \mathbb{E}\left[\left(\partial_{k} f\right)\left(X_{1}, \ldots, X_{d}\right)\right] .
$$

Proof. If the covariance matrix $C$ of the $X_{i}$ 's is $I$, then the result follows from a one-dimensional integration by parts. For a more general covariance matrix $C$, introduce a standard Gaussian vector $Y$, so that as $X \stackrel{\text { law }}{=} A Y$ for $A:=C^{1 / 2}$ and the function

$$
g\left(y_{1}, \ldots, y_{d}\right):=f \circ A\left(y_{1}, \ldots, y_{d}\right)
$$


Then by the $C=I$ case, we have

$$
\begin{aligned}
\mathbb{E}\left[X_{i_{0}} f\left(X_{1}, \ldots, X_{d}\right)\right] & =\sum_{j} A_{i_{0} j} \mathbb{E}\left[Y_{j} g\left(Y_{1}, \ldots, Y_{d}\right)\right] \\
& =\sum_{j} A_{i_{0} j} \mathbb{E}\left[\left(\partial_{j} g\right)\left(Y_{1}, \ldots, Y_{d}\right)\right] \\
& =\sum_{j} A_{i_{0} j} \sum_{k} A_{k j} \mathbb{E}\left[\left(\partial_{k} f\right)\left(X_{1}, \ldots, X_{d}\right)\right] \\
& =\sum_{k} C_{i_{0} k} \mathbb{E}\left[\left(\partial_{k} f\right)\left(X_{1}, \ldots, X_{d}\right)\right] .
\end{aligned}
$$

\section{Appendix B. Concentration}

The following lemma can be found for example in ([2], Sect. 4.4.1).

Lemma B.1. Let $X=\left(X_{1}, \ldots, X_{d}\right)$ be a standard real Gaussian vector and $f: \mathbb{R}^{d} \rightarrow \mathbb{R}$ be a $\mathcal{C}^{1}$ function with gradient $\nabla f$. Then we have

$$
\operatorname{Var}(f(X)) \leq \mathbb{E}\|\nabla f(X)\|^{2},
$$

where $\|\cdot\|$ denotes the standard Euclidian norm.

Besides, if $f$ is $k$-Lispchitz, then for any $t>0$, we have

$$
\mathbb{P}(|f(X)-\mathbb{E} f(X)| \geq t) \leq 2 \mathrm{e}^{-\frac{t^{2}}{2 k^{2}}} .
$$

To apply this lemma, we shall use the following lemma. All matrix spaces, here, are endowed with the norm $\sqrt{\operatorname{Tr} M M^{*}}$.

Lemma B.2. Let $f$ be a real (resp. complex) function on $\mathbb{R}^{+}$such that $x \mapsto f\left(x^{2}\right)$ is c-Lipschitz. Then the functions $\varphi, \psi$, defined on the set of $p \times n$ complex matrices by $\varphi(X)=f\left(X X^{*}\right)$ and $\psi(X)=f\left(X^{*} X\right)$ are c-Lipschitz (resp. 2c-Lipschitz).

Proof. The complex case is directly deduced from the real one by writing $f=\Re(f)+\Im(f)$. So let us suppose that $f$ is real-valued. Let $g: x \mapsto f\left(x^{2}\right)$ and $N:=p+n$. We know, by ([7], Lemma A.2), that the extension of $g$ to the set of $N \times N$ Hermitian matrices is $c$-Lipschitz. Then, the conclusion follows from the fact that for any $p \times n$ complex matrix $X, \varphi(X)$ and $\psi(X)$ are the respective $p \times p$ upper-left corner and $n \times n$ lower-right corner of the $N \times N$ matrix $g(M)$, with

$$
M:=\left(\begin{array}{cc}
0 & X \\
X^{*} & 0
\end{array}\right) .
$$

\section{Appendix C. Nonnegative Matrices}

The results stated here can be found in $[14,15]$.

Lemma C.1 (Nonnegative matrices and dominant eigenvectors). If $A \in \mathbb{R}_{+}^{n \times n}$ is nonnegative (resp., positive), then $\rho(A)$ is an eigenvalue of $A$ having an eigenvector with nonnegative (resp., positive) entries.

Lemma C.2 (Spectral radii). Let $A, B \in \mathbb{R}^{n \times n}$ be such that $\left|A_{i j}\right| \leq B_{i j}$ for all $1 \leq i, j \leq n$. Then, with $\rho$ the spectral radius,

$$
\rho(A) \leq \rho(B)
$$


Lemma C.3 (Cauchy-Schwarz for spectral radii (adapted from [15], Lem. 5.7.9)). Let $A, B \in \mathbb{R}_{+}^{n \times n}$ be non negative matrices and $C \in \mathbb{R}^{n \times n}$ be such that $C_{i j} \leq \sqrt{A_{i j} B_{i j}}$. Then,

$$
\rho(C) \leq \sqrt{\rho(A) \rho(B)}
$$

Besides, if, for each $i$, either both the ith row and the ith column of $C$ are null or there exists $j$ such that $C_{i j}<\sqrt{A_{i j} B_{i j}}$, then the inequality is strict.

\section{REFERENCES}

[1] O. Ajanki, L. Erdös and T. Kruger Quadratic vector equations on complex upper half-plane (2015).

[2] G. Anderson, A. Guionnet and O. Zeitouni, An Introduction to Random Matrices. Vol. 118 of Cambridge Studies Advanced Math. (2009).

[3] Z.D. Bai and J.W. Silverstein, No eigenvalues outside the support of the limiting spectral distribution of large dimensional sample covariance matrices. Ann. Probab. (1998) 26 316-345.

[4] J. Baik, G. Ben Arous and S. Péché, Phase transition of the largest eigenvalue for nonnull complex sample covariance matrices. Ann. Probab. 33 (2005) 1643-1697.

[5] F. Benaych-Georges and R.N. Rao, The eigenvalues and eigenvectors of finite, low rank perturbations of large random matrices. Adv. Math. (2011) 227 494-521.

[6] F. Benaych-Georges and R.N. Rao, The singular values and vectors of low rank perturbations of large rectangular random matrices. J. Multivariate Anal. 111 (2012) 120-135.

[7] M. Capitaine, Additive/multiplicative free subordination property and limiting eigenvectors of spiked additive deformations of Wigner matrices and spiked sample covariance matrices. J. Theor. Probab. 26 (2013) 595-648.

[8] F. Chapon, R. Couillet, W. Hachem and X. Mestre, The outliers among the singular values of large rectangular random matrices with additive fixed rank deformation. Markov Process. Relat. Fields 20 (2014) 183-228.

[9] R. Couillet and W. Hachem, Analysis of the limiting spectral measure of large random matrices of the separable covariance type. Random Matrices: Theory Appl. 3 (2014) 1450016.

[10] R. Couillet and F. Benaych-Georges, Kernel spectral clustering of large dimensional data. Electron. J. Stat. 10 (2016) 13931454 .

[11] R. Couillet, M. Debbah and J.W. Silverstein, A deterministic equivalent for the analysis of correlated MIMO multiple access channels. IEEE Trans. Inform. Theory 57 (2011) 3493-3514.

[12] L. Erdös, B. Schlein and H.-T. Yau, Semicircle law on short scales and delocalization of eigenvectors for Wigner random matrices. Ann. Prob. 37 (2009).

[13] T. Hastie, R. Tibshirani and J. Friedman, The elements of statistical learning. Data mining, inference, and prediction. Springer Series in Statistics, 3nd edition. Springer, New York (2009).

[14] R.A. Horn and C.R. Johnson, Matrix Analysis. Cambridge University Press (2013).

[15] R.A. Horn and C.R. Johnson, Topics in Matrix Analysis. Cambridge University Press (1991).

[16] G. James, D. Witten, T. Hastie and R. Tibshirani, An introduction to statistical learning. With applications in R. Vol. 103 of Springer Texts in Statistics. Springer, New York (2013).

[17] I.M. Johnstone, On the distribution of the largest eigenvalue in principal components analysis. Ann. Statist. 29 (2001) 295327.

[18] A. Kammoun, M. Kharouf, W. Hachem and J. Najim, A central limit theorem for the SINR at the LMMSE estimator output for large-dimensional signals. IEEE Trans. Inform. Theory 55 (2009) 5048-5063.

[19] R. Kannan and S. Vempala, Spectral algorithms. Found. Trends Theoret. Comput. Sci. 4 (2009) 157-288.

[20] V. Kargin, A concentration inequality and a local law for the sum of two random matrices. Probab. Theory Related Fields $\mathbf{1 5 4}$ (2012) 677-702.

[21] P. Loubaton and P. Vallet, Almost sure localization of the eigenvalues in a Gaussian information plus noise model. Applications to the spiked models. Electron. J. Probab. 16 (2011) 1934-1959.

[22] U. von Luxburg, A tutorial on spectral clustering. Stat. Comput. 17 (2007) 395-416.

[23] V.A. Marcenko and L.A. Pastur, Distribution of eigenvalues for some sets of random matrices. Sb. Math. 1 (1967) $457-483$.

[24] J.W. Silverstein and S. Choi, Analysis of the limiting spectral distribution of large dimensional random matrices. J. Multivariate Anal. 54 (1995) 295-309. 